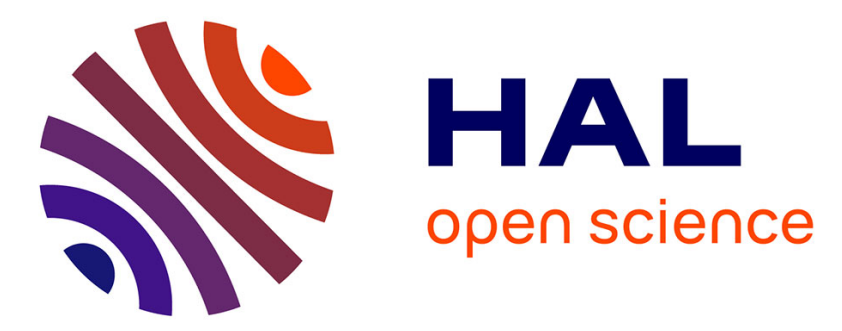

\title{
Organocatalytic aza-Michael Reaction to 3-Vinyl-1,2,4-triazines as a Valuable Bifunctional Platform
}

Floris Buttard, Clément Berthonneau, Marie-Aude Hiebel, Jean-François Brière, Franck Suzenet

\section{To cite this version:}

Floris Buttard, Clément Berthonneau, Marie-Aude Hiebel, Jean-François Brière, Franck Suzenet. Organocatalytic aza-Michael Reaction to 3-Vinyl-1,2,4-triazines as a Valuable Bifunctional Platform. Journal of Organic Chemistry, 2019, 84 (6), pp.3702-3714. 10.1021/acs.joc.9b00141 . hal-02197289

HAL Id: hal-02197289

https://hal-normandie-univ.archives-ouvertes.fr/hal-02197289

Submitted on 21 Aug 2020

HAL is a multi-disciplinary open access archive for the deposit and dissemination of scientific research documents, whether they are published or not. The documents may come from teaching and research institutions in France or abroad, or from public or private research centers.
L'archive ouverte pluridisciplinaire HAL, est destinée au dépôt et à la diffusion de documents scientifiques de niveau recherche, publiés ou non, émanant des établissements d'enseignement et de recherche français ou étrangers, des laboratoires publics ou privés. 


\section{Organocatalytic aza-Michael Reaction to 3-Vinyl-1,2,4-Triazines as a Valuable Bifunctional Platform}

Floris Buttard, ${ }^{\mathrm{a}}$ Clément Berthonneau, ${ }^{\mathrm{b}}$ Marie-Aude Hiebel, ${ }^{\mathrm{a}}$ Jean-François Brière, ${ }^{\mathrm{b} *}$ and Franck Suzenet ${ }^{\text {a } *}$

a Université d'Orléans, CNRS, ICOA, UMR 7311, 45067 Orléans, France. Phone: (+33)-2-3849-4580; E-mail: franck.suzenet@univ-orleans.fr

b Normandie Univ, UNIROUEN, INSA Rouen, CNRS, COBRA, 76000 Rouen, France. Phone: (+33)-2-3552-2464; e-mail: jean-francois.briere@insa-rouen.fr.

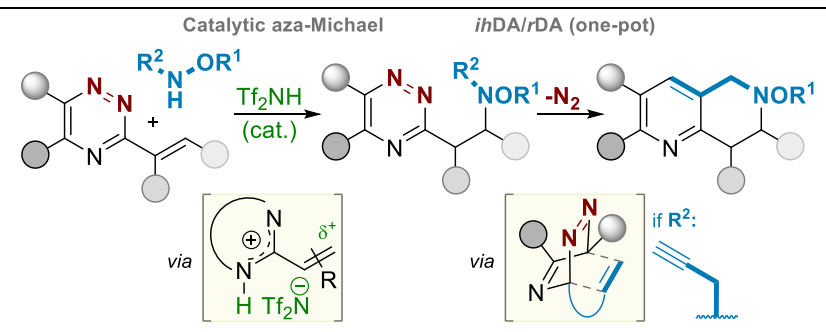

An unprecedented catalytic aza-Michael addition to substituted 3-vinyl-1,2,4-triazines, as original bifunctional platforms for domino conjugate addition-ihDA/rDA reaction, was achieved using the highly acidic triflimide as organocatalyst. Based on the use of alkoxyamine nucleophiles, this sequence not only highlights a rare example of catalytic azaMichael reaction to alkenylazaarenes but proved to be useful for the elaboration of an array of biorelevant tetrahydro-[1,6]-naphthyridines.

Medicinal chemistry and chemical biology are currently seeking original heterocyclic scaffolds as biological tools. Accordingly, the development of new synthetic methodologies aiming at exploring chemical space, while increasing molecular diversity and complexity, is a current concern in organic synthesis. ${ }^{1}$ The construction of fused non-aromatic/heteroaromatic bicycles has emerged as a promising strategy furnishing unprecedented potent bioactive 
molecules. ${ }^{2}$ The tuning of the selectivity and properties such as solubility was allowed by changing aromatic polycyclic drug candidates into partially saturated ones. ${ }^{3}$ Additionally, by escaping the flatland, the presence of $\mathrm{sp}^{3}$ hybridized carbons and chiral stereocenters within bioactive compounds maximizes the on-target profile, which decreases the risk of toxicity issues in the clinic setting. ${ }^{4}$ However, the introduction of substituents on the saturated moiety of fused non-aromatic/heteroaromatic bicycles remains a challenging synthetic task.

We recently introduced 3-vinyl-1,2,4-triazines 1 behaving as (1) an original Michael acceptor belonging to the alkenylazaarene series, ${ }^{5,6}$ and (2) an aza-diene during the subsequent intramolecular domino inverse-electron-demand hetero-Diels-Alder/retro-Diels-Alder $(i h \mathrm{DA} / r \mathrm{DA})$ reaction (Scheme 1a).,8 This versatile platform $\mathbf{1}$ gave access to nonaromatic/heteroaromatic bicycles ${ }^{8}$ displaying a privileged pyridine scaffold ${ }^{9,10,11}$ flanked by a saturated carba ${ }^{8 c}$ and thia-ring. ${ }^{8 a}$ In a recent aza-Michael based extension $(\mathrm{Nu}=\mathrm{N})$, platform 1 furnished a novel entry to tetrahydro-[1,6]-naphthyridines (Scheme 1b). ${ }^{8 b}$

Scheme 1. Context of the investigation

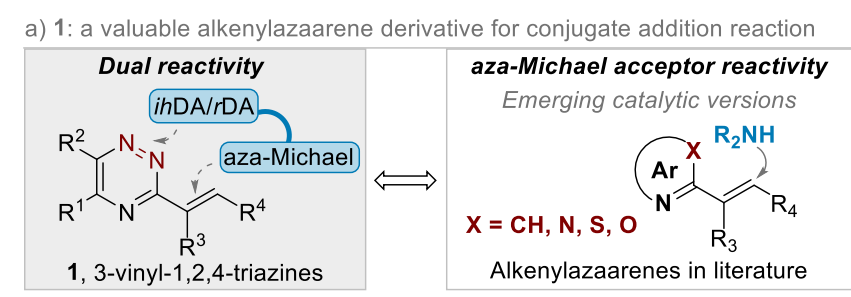

b) 1: a dual-platform for non-aromatic/heteroaromatic bicycle construction

$$
\begin{aligned}
& \text { Tetrahydro-[1,6]-naphthyridines (literature) } \\
& \text { - Limited methods for } R^{3-5} \neq \mathrm{H}
\end{aligned} \begin{aligned}
& \text { From 1 via an aza-Michael }-\mathrm{R}^{3-5} \neq \mathrm{H} \\
& \text { - Marked limitation for } R^{3} \text { and } R^{4} \text { (essentially } \mathrm{Me} \text { ) } \\
& \text { - Non-catalytic aza-Michael }
\end{aligned}
$$

c) First catalytic aza-Michael reaction to 3-vinyl-1,2,4-triazines 1 (this work)

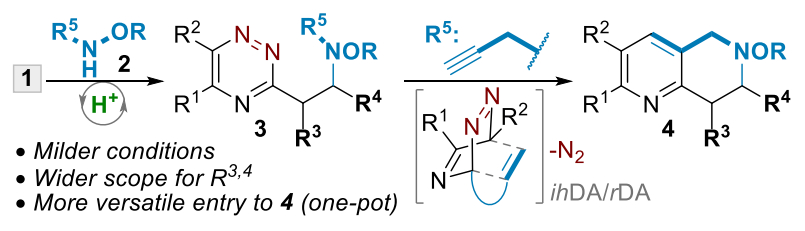

While this preliminary investigation afforded various derivatives functionalized on the pyridine ring, only a few substituents (i.e. methyl) were distributed on the saturated moiety. 
Indeed, the 1,4-conjugate addition to 1 proved to be more challenging when the vinyl moiety is substituted. The reaction proceeded with high temperature or an excess of Lewis acid which poses compatibility issues with the subsequent (ihDA/rDA) reaction. ${ }^{8 b}$ To overcome this limited reactivity, an improved catalytic aza-Michael reaction was sought which would also provide a new route to diversely substituted tetrahydro-[1,6]-naphthyridines. Importantly, despite the importance of this non-aromatic/heteroaromatic bicycles in medicinal chemistry, the reported syntheses addressed in some cases the substitution at $\mathrm{C} 5$ position $\left(\mathrm{R}^{5}\right),{ }^{12}$ but the strategies allowing the introduction of $\mathrm{R}^{3-4}$ remains rather limited. ${ }^{13}$

The conjugate addition reactions to alkenylazaarenes have elicited useful synthetic applications in organic synthesis, ${ }^{5}$ but the catalytic versions appeared only recently. Apart from metal-based catalysis, ${ }^{5 a}$ a few elegant organocatalytic approaches, ${ }^{14,15,16}$ emerged to activate vinyl-derived pyridines $(X=C H)$, diazole $(X=N)$, -oxazole $(X=O)$ and thiazole $(X$ $=\mathrm{S}$ ) compounds toward 1,4-conjugate addition reaction (Scheme 1a). Meanwhile, the catalytic aza-Michael reaction to alkenylazaarenes has remained elusive, ${ }^{5,17,18}$ until the recent asymmetric catalytic 1,4-conjugated addition of pyrazole-heterocycle derivatives to alkenylbenzimidazoles $(\mathrm{X}=\mathrm{N})$ described by the group of Terada. ${ }^{14 \mathrm{c}}$ Recently, Watson and colleagues reported an original catalytic and enantioselective aza-Michael-protonation reaction of aniline-derivatives to $\alpha$-substituted vinyl-pyridines and derivatives $\left(R^{3}=\operatorname{Ar}, R^{4}=\right.$ $\mathrm{H}, \mathrm{X}=\mathrm{CH}, \mathrm{S}, \mathrm{O})$ in the presence of phosphoric acids. ${ }^{19}$

3-Vinyl-1,2,4-triazines $\mathbf{1}$ are novel platforms in this field of catalytic Michael reactions. ${ }^{20}$ Herein, we are pleased to disclose an original Brønsted acid catalyzed aza-Michael reaction of alkoxyamine nucleophiles 2 to variously substituted 3-vinyl-1,2,4-triazines 1 (Scheme 1c). This organocatalytic approach not only overrides the previous limitation regarding the access of adducts 3 but also expands the scope for the subsequent construction of tetrahydro-[1,6]- 
naphthyridines $\mathbf{4}$ diversely substituted on the saturated ring thanks to a straightforward $i h \mathrm{DA} / r \mathrm{DA}$ sequence.

We previously observed the conjugate addition reaction of $N$-methylbenzylamine 2 a to unsubstituted 3-vinyl-1,2,4-triazine in $\mathrm{MeOH}$ at $30{ }^{\circ} \mathrm{C}$ to give adduct 3a in quantitative yield (Table 1 , entry 1$){ }^{8 b}$

\section{TABLE 1. Proof of principle ${ }^{a}$}

$$
\mathbf{1 a}\left(\mathrm{R}^{1}=\mathrm{H}\right), \mathbf{1 b}\left(\mathrm{R}^{1}=\mathrm{Me}\right) \quad 2 \quad \frac{\mathrm{R}^{1}}{30^{\circ} \mathrm{C}, 24 \mathrm{~h}}
$$

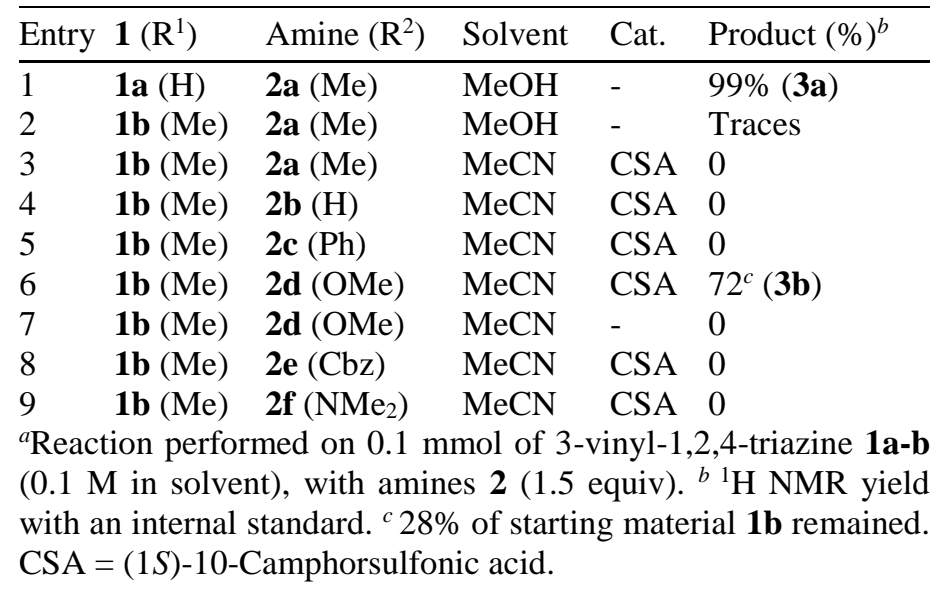

However, this smooth reactivity was not adapted to more hindered 3-vinyl-1,2,4-triazines such as $\mathbf{1 b}$ giving only traces of the corresponding adduct (entry 2). In line with the use of phosphoric acid in this field, ${ }^{14 \mathrm{~b}-\mathrm{d}, 19}$ we foresaw that a more effective organocatalytic activation of the Michael acceptor $\mathbf{1 b}$ would take place with a sufficiently strong acid catalyst through protonation of the triazine ring of $\mathbf{1 b}$. However, a Brønsted acid organocatalyst in the presence of a moderately basic triazine moiety may competitively lead to a non-productive protonation of either the amine $\mathbf{2}$ or the aza-Michael adduct 3. First of all, we used camphor sulfonic acid (CSA, $20 \mathrm{~mol} \%$ ) as catalyst in acetonitrile with benzylamines 2a-d bearing different $N$-functional groups (entries 3-6). While no transformation was observed with amines 2a-c (entries 3-5), which possess carbon-based substituents, the methoxyamine 
nucleophile 2d (entry 6) led to the desired aza-Michael adduct 3b in a promising $72 \%$ NMR yield. The CSA catalyst appeared to be essential for this transformation (entry 7), as well as the NHOMe motif since no aza-Michael reaction took place with NHCbz $\mathbf{2 e}$ or hydrazine $\mathbf{2 f}$ nucleophiles (entries 8-9). Although the exact mechanism deserves further investigations, it can be assumed that the moderate basicity of the methoxyamine $\mathbf{2 d}$ minimizes its competitive (non-productive) protonation versus the protonative-activation of triazine $\mathbf{1 b}$ while keeping a sufficiently nucleophilic character (in comparison to RNHCbz $2 \mathbf{e}$ for instance) to secure the conjugate addition process. 
TABLE 2. Catalyst screening ${ }^{a}$

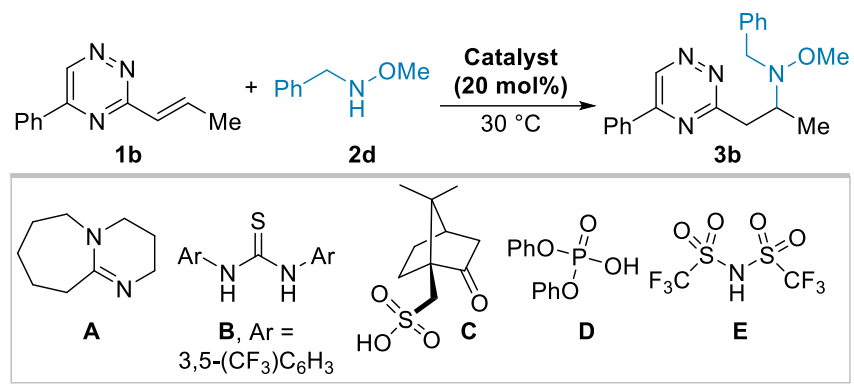

\begin{tabular}{|c|c|c|c|c|}
\hline Entry & $\begin{array}{l}\text { Catalyst } \\
(\mathrm{mol} \%)\end{array}$ & Time (h) & Solvent & Product $\mathbf{3 b}(\%)^{b}$ \\
\hline 1 & $\mathbf{A}(20)$ & 24 & $\mathrm{MeCN}$ & 0 \\
\hline 2 & B (20) & 24 & $\mathrm{MeCN}$ & 0 \\
\hline 3 & C (20) & 24 & $\mathrm{MeCN}$ & 72 \\
\hline 4 & D (20) & 24 & $\mathrm{MeCN}$ & 45 \\
\hline 5 & $\mathbf{E}(20)$ & 24 & $\mathrm{MeCN}$ & $90(81)^{c}$ \\
\hline 6 & $\mathbf{E}(20)$ & 3 & $\mathrm{MeCN}$ & 54 \\
\hline 7 & $\mathbf{E}(20)$ & 3 & toluene & 58 \\
\hline 8 & $\mathbf{E}(20)$ & 3 & $\mathrm{THF}$ & 15 \\
\hline 9 & $\mathbf{E}(20)$ & 3 & $\mathrm{CHCl}_{3}$ & 49 \\
\hline 10 & $\mathbf{E}(20)$ & 3 & $\mathrm{Et}_{2} \mathrm{O}$ & 58 \\
\hline 11 & $\mathbf{E}(20)$ & 24 & toluene & 99 \\
\hline 12 & $\mathbf{E}(10)$ & 24 & toluene & 89 \\
\hline 13 & $\mathbf{E}(5)$ & 24 & toluene & 28 \\
\hline \multicolumn{5}{|c|}{$\begin{array}{l}{ }^{a} \text { Reaction performed on } 0.1 \mathrm{mmol} \text { of } 3 \text {-vinyl-1,2,4-triazine } \\
\mathbf{1 b}\left(0.1 \mathrm{M} \text { in solvent), with amine } \mathbf{2 d}(1.5 \text { equiv }) .{ }^{b}{ }^{1} \mathrm{H} \mathrm{NMR}\right. \\
\text { yield with an internal standard. }{ }^{c} \text { Isolated yield after silica } \\
\text { gel column chromatography. }\end{array}$} \\
\hline
\end{tabular}

With this preliminary result in hand, different kind of organocatalysts (20 mol\%) were subsequently evaluated with methoxybenzylamine nucleophile 2d (Table 2, see supporting information for further details). It was demonstrated that neither Brønsted bases such as DBU A (entry 1), nor Schreiner's type catalyst B (entry 2), as a hydrogen bond donor species, were capable to provide any Michael-adduct. Brønsted acid catalysts, as expected, allowed the formation of adduct $\mathbf{3 b}$ (entries 3-5). Interestingly, the efficiency of the reaction was directly correlated to the acidity of the catalyst. Lower activity was observed with phosphoric acid D (45\%, entry $6, \mathrm{p} K_{\mathrm{a}} \approx 3.8$ in DMSO) with regard to CSA $\mathbf{C}\left(72 \%\right.$, entry $5, \mathrm{p} K_{\mathrm{a}} \approx 1.6$ in DMSO). Eventually, the highly acidic triflimide $\mathbf{E}$ proved to be the more potent organocatalyst (entry 5), ${ }^{21,22}$ allowing the formation of product $\mathbf{3 b}$ with an excellent $90 \%$ 
NMR yield after 24 hours reaction (81\% isolated yield). Solvent optimization (entries 6-11) revealed that diethyl ether (entry 10), as a less polar solvent, gave the best conversions together with toluene in 3 hours (entry 7), which secured an excellent 99\% NMR yield after 24 hours (entry 11). In these conditions, the triflimide catalyst $\mathbf{E}$ loading was decreased to 10 mol\% with a minor impact on efficiency (entry $12,89 \%$ yield) whereas a limit was reached with 5 mol\% of $\mathbf{E}$ (entry 13, 28\% yield after 24 hours).

We subsequently evaluated the scope of the optimal conditions $\left(20\right.$ mol\% of $\mathrm{HNTf}_{2} \mathbf{E}$ in toluene for 24 hours) by focusing on vinyl-triazines 1 with various substituents on the vinyl moiety (Figure 1). The precursors 1 were readily synthesized by means of a Liebeskind-Srogltype coupling reaction ${ }^{23}$ between alkenyl-boronic acid derivatives and 3-thiomethylfuntionalized triazines ${ }^{24}$ in line with our previous developments. ${ }^{8}$ The conjugated addition reaction proceeded uneventfully with substrates possessing $\alpha$ - or $\beta$-substituted alkenes giving rise to adducts $\mathbf{3 b}$ and $\mathbf{3 c}$ with methyl group in $97 \%$ and $77 \%$ isolated yields, respectively. For comparison, we previously showed that the 2-propenyl platform $3 \mathbf{c}$ required a large excess of $\mathrm{BF}_{3} . \mathrm{OEt}_{2}$ at refluxing THF to undergo the aza-Michael reaction. ${ }^{8 \mathrm{~b}}$ Interestingly, an alcohol pendant was also tolerated with the catalytic conditions (3d, 85\%). More hindered $\beta$ - $n$-pentyl 3e (79\%), -cyclopropyl 3f (46\%) and -benzyl 3g (39\%) were also good substrates even if they required longer reaction times for completion (72-96 h). Due to instability issues on silica-gel column chromatography, $\mathbf{3 g}$ was isolated in a moderate isolated $39 \%$ yield although $80 \%$ was estimated by ${ }^{1} \mathrm{H}$ NMR with an internal standard. Limitations were observed with more hindered cyclohexyl (3h) or phenyl (3i) derivatives. It was also proven that the reaction occurred efficiently with triazines flanked by various substituents such as 5-methyl (3j-73\%), 5-CF $3(3 k-79 \%)$ or 5,6-diMe (3l-87\%). The more challenging 5,6-unsubstituted triazine $\mathbf{1 m}$, as a less stable platform, was also transformed into $\mathbf{3 m}$ with a promising $39 \%$ yield. The azaMichael reaction was also extrapolated either to propargylamine nucleophiles $\mathbf{2 g - h}$ and 
primary $\mathrm{BnONH}_{2} \mathbf{2} \mathbf{i}$ to furnish the corresponding adducts $\mathbf{3 n}-\mathbf{3 p}$ in very good yields (81$91 \%)$, and up to $1.1 \mathrm{mmol}$ scale for product $\mathbf{3 n}$ (88\% isolated yield).

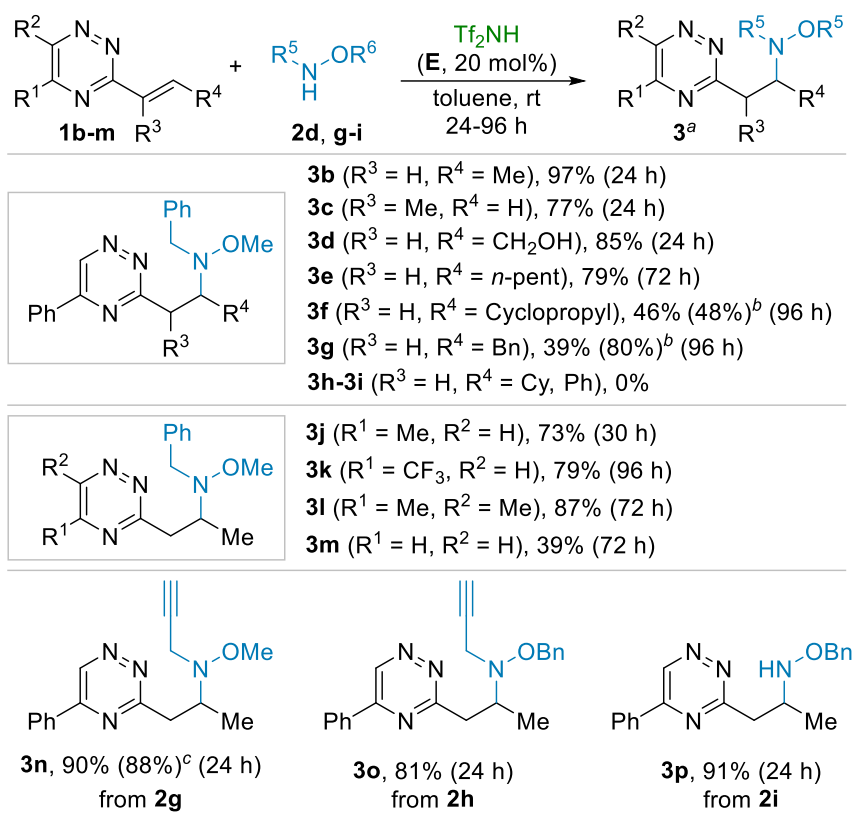

Figure 1. Scope and limitations of the aza-Michael reaction. ${ }^{a}$ Reaction performed on $0.1 \mathrm{mmol}$ of 3 -vinyl1,2,4-triazines $1(0.1 \mathrm{M})$, with amines 2 (1.5 equiv) in 24 hours. Isolated yield after silica gel column chromatography. ${ }^{b}{ }^{1} \mathrm{H}$ NMR yield determined by an internal standard into bracket. ${ }^{c}$ Carried out on $1.1 \mathrm{mmol}$ scale.

In order to address the synthesis of tetrahydro-[1,6]-naphthyridines such as $\mathbf{4 a}$, we investigated the $i h \mathrm{DA} / r \mathrm{DA}$ sequence with the model substrate $\mathbf{3 q}$ (Figure 2). The heteroDiels-Alder reaction proceeded rapidly in 2 hours when $180{ }^{\circ} \mathrm{C}$ heating was carried out conveniently upon microwave (MW) irradiation. These conditions furnished $\mathbf{4 a}$ in a complete conversion and high $95 \%$ yield up to $1.7 \mathrm{mmol}$ scale. Importantly, it was observed that the pyridine product $\mathbf{4 a}$, or an intermediate derived thereof, were unstable upon longer heating time, as evidenced by a $79 \%$ yield obtained after 3 hours at $180{ }^{\circ} \mathrm{C}$ (Figure 2), showing the importance of mastering this cycloaddition step. 


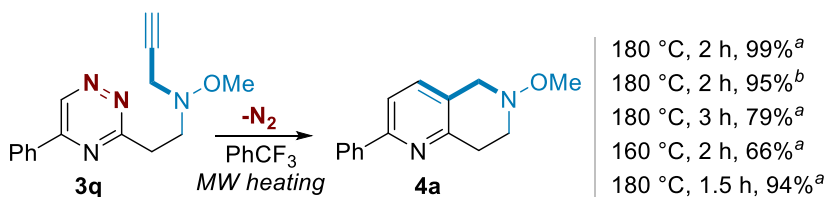

Figure 2. Optimization and prospective of the domino ihDA/rDA reaction. Conditions: starting material 3q in $\mathrm{CF}_{3} \mathrm{Ph}(0.1 \mathrm{M})$ was heated under microwave irradiation (MW). ${ }^{a}{ }^{1} \mathrm{H}$ NMR yield determined by an internal standard. ${ }^{b}$ Isolated yield on $1.7 \mathrm{mmol}$ scale after column chromatography.

Given this stability issue, a sequential aza-Michael reaction with a complete transformation followed by a well-controlled $i h \mathrm{DA} / r \mathrm{DA}$ sequence upon heating in 2 hours was conducted in order to synthesize novel tetrahydro-[1,6]-naphthyridines 4 (Figure 3). ${ }^{8 b}$

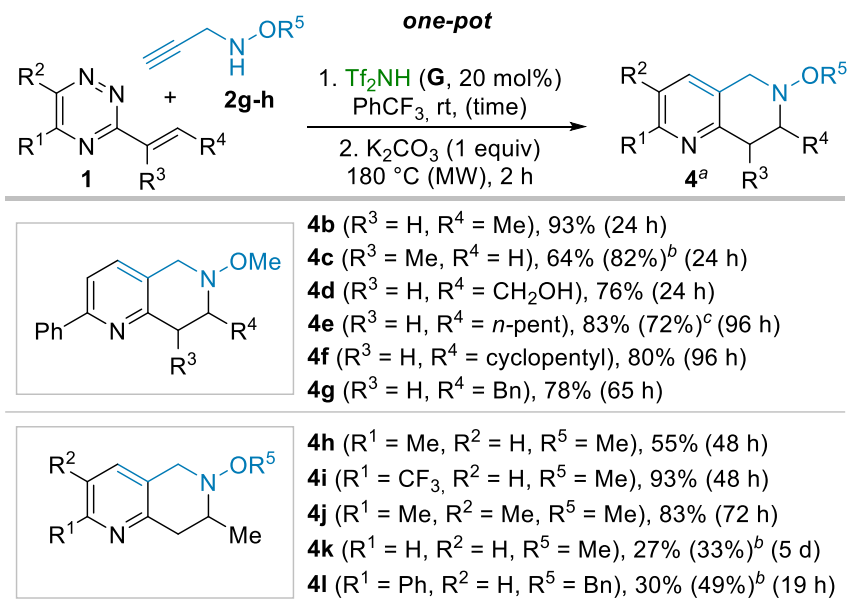

Figure 3. Scope and limitations of the domino sequential aza-Michael-ihDA/rDA reaction. ${ }^{a}$ Reaction performed on $0.3 \mathrm{mmol}$ of 3-vinyl-1,2,4-triazines $\mathbf{1}(0.1 \mathrm{M})$, with propargylamines $\mathbf{2 g - h}$ (1.5 equiv) in 24 hours. Isolated yield after silica gel column chromatography (over two steps). ${ }^{b}{ }^{1} \mathrm{H}$ NMR yield determined by an internal standard into bracket. ${ }^{c}$ Carried out on $1 \mathrm{mmol}$ scale for 90 hours.

This one-pot process was carried out in trifluorotoluene, as a microwave friendly solvent. Early attempts demonstrated that the presence of triflimide $\mathbf{E}$ led to major decomposition events during the cycloaddition process at high temperature. To prevent this acid promoted degradation, $\mathrm{K}_{2} \mathrm{CO}_{3}$ (1 equivalent) was added before the cycloaddition step allowing an efficient one-pot process (see experimental section) towards the formation of a series of novel 
tetrahydro-[1,6]-naphthyridines $\mathbf{4 b}$-j flanked by various substituents as depicted in Figure 3, with good to excellent overall yields (55-93\%). Even the more challenging 5,6-unsubstituted triazine starting material $\mathbf{1 m}$ gave the corresponding product $\mathbf{4 k}$ in $27 \%$ yield in two steps. Interestingly, the $\mathrm{N}$-OBn derived product $\mathbf{4 l}$ (via adduct $\mathbf{3 n}$ ) was also accessible albeit in a moderate $30 \%$ isolated yield, likely due to decomposition events.

Finally, in order to further exploit the new and valuable pyridine derivatives $\mathbf{4}$ in medicinal chemistry programs for example, we turned our attention to converting representative methoxyamine derived products $\mathbf{4}$ into the secondary amines $\mathbf{5}$. A straightforward $\mathrm{N}-\mathrm{O}$ bond cleavage took place in the presence of $\mathrm{SmI}_{2}$, according to Brandi's procedure, ${ }^{25}$ opening the access to $\mathrm{NH}$-tetrahydro-[1,6]-naphthyridines 5a-c with yields ranging from $70 \%$ to $84 \%$ (Scheme 2).

\section{Scheme 2. $\mathrm{N}-\mathrm{O}$ bond cleavage by $\mathrm{SmI}_{2}$}

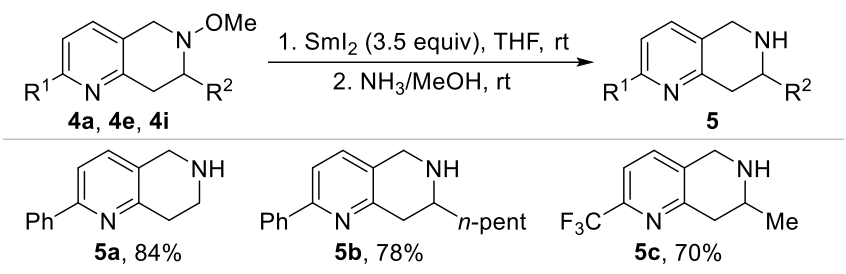

In summary, the first catalytic aza-Michael reaction to substituted 3-vinyl-1,2,4-triazines $\mathbf{1}$ was achieved by means of the strong triflimide acid $\mathbf{E}$ as organocatalyst in the presence of alkoxyamines 2. This approach shows a rare example of catalytic aza-Michael reaction to alkenylazaarene derivatives, and markedly broadens the scope of triazine aza-Michael adducts 3 accessible from various substituents on the vinyl moiety. Thanks to the unique dual reactivity of platform $\mathbf{1}$, the subsequent $i h \mathrm{DA} / r \mathrm{DA}$ reaction took place in a convenient onepot fashion from the corresponding propargylamines $\mathbf{2}$ allowing the elaboration of various biorelevant tetrahydro-[1,6]-naphthyridine derivatives 4. Investigations towards an enantioselective version are currently in progress. 


\section{Experimental Section}

\section{General Remarks}

Unless otherwise specified, all reagent-grade chemicals and solvents commercially available were used without further purification. The reactions were monitored by thin-layer chromatography (TLC) using aluminum sheets coated with silica gel 60 F254. Flash column chromatography was carried out using silica gel $60 \AA(0.04-0.06 \mathrm{~mm})$. Solvents mentioned as dry were purified with a dry station GT S100 immediately prior to use. Microwave irradiation experiments were performed on a Monowave 300 single-mode microwave reactor (Anton Paar GmbH) using standard 10 or 30 mL Pyrex vessels (G10 or G30) equipped with Tefloncoated magnetic stir bar and closed with snap cap and silicone septum. The microwave apparatus has to be equipped with a safety pressure shutoff. Experiments carried out on 0.3 mmol scale of triazine in a $10 \mathrm{~mL}$ vial can generate 6-9 bars of pressure. NMR spectra were recorded with a $250 \mathrm{MHz}\left({ }^{1} \mathrm{H}: 250 \mathrm{MHz}\right.$ and $\left.{ }^{13} \mathrm{C}: 63 \mathrm{MHz}\right)$ or $400 \mathrm{MHz}\left({ }^{1} \mathrm{H}: 400 \mathrm{MHz}\right.$ and

${ }^{13} \mathrm{C}$ : $\left.100.7 \mathrm{MHz}\right)$ Bruker spectrometer. Unless noted the spectra were recorded at $25{ }^{\circ} \mathrm{C}$. Chemical shifts are given in parts per million (ppm) from tetramethylsilane (TMS), calibrated to the residual solvent peak. Coupling constants " $J$ " are expressed in hertz (multiplicity: $\mathrm{s}=$ singlet, br $\mathrm{s}=$ broad singlet, $\mathrm{d}=$ doublet, $\mathrm{dd}=$ double doublet, $\mathrm{dt}=$ double triplet, $\mathrm{t}=$ triplet, $\mathrm{q}$ $=$ quartet, $\mathrm{p}=$ pentuplet, $\mathrm{h}=$ hexuplet, hept $=$ heptuplet, $\mathrm{m}=$ multiplet $\ldots)$. The ${ }^{13} \mathrm{C}$ NMR peak assignments have been confirmed using Heteronuclear Multiple Bond Correlation (HMBC) and Distortionless Enhancement by Polarization Transfer (DEPT-135) experiments. Highresolution accurate mass measurements (HRAM) were performed on a Bruker maXis mass spectrometer by the "Fédération de Recherche" ICOA/CBM (FR2708) platform using an electrospray ionization source (ESI) in positive mode and a time-of-flight (TOF) analyzer. Melting points were measured in open capillary tubes. The infrared spectra were recorded on a FT-IR Thermo Scientific Nicolet iS10 and maximum absorption wavenumbers $v_{\max }$ are 
given in $\mathrm{cm}^{-1}$.

3-(methylthio)-1,2,4-triazine, ${ }^{26}$ 3-(methylthio)-5-(trifluoromethyl)-1,2,4-triazine, ${ }^{10 \mathrm{e}}$ N-Benzyl-O-methylhydroxylamine $\mathbf{2 d},{ }^{27}$ benzyl N-benzylcarbamate $\mathbf{2 e}^{28}$ and 2-benzyl-1,1dimethylhydrazine $\mathbf{2} \mathbf{f}^{29}$ were synthetized according to literature procedures. 3-(methylthio)-5phenyl-1,2,4-triazine,${ }^{6}$ 5,6-dimethyl-3-(methylthio)-1,2,4-triazine, ${ }^{8 \mathrm{~b}} 3$-vinyl-1,2,4-triazine $\mathbf{1 a},{ }^{6}$ (E)-5-phenyl-3-(prop-1-en-1-yl)-1,2,4-triazine $\quad \mathbf{1 b}{ }^{8 \mathrm{~b}} \quad$ 5-phenyl-3-(prop-1-en-2-yl)-1,2,4triazine $\mathbf{1} \mathbf{c}^{8 \mathrm{~b}}$ and (E)-3-(5-phenyl-1,2,4-triazin-3-yl)prop-2-en-1-ol $\mathbf{1 d}^{8 \mathrm{~b}}$ were synthetized as previously described.

\section{5-methyl-3-(methylthio)-1,2,4-triazine}

A solution of S-methylthiosemicarbazide iodohydrate $(11.7 \mathrm{~g}, 50.2 \mathrm{mmol})$ in $50 \mathrm{~mL}$ of water at $0{ }^{\circ} \mathrm{C}$ was added to a stirred solution of pyruvaldehyde (40\% in water, $9.2 \mathrm{~mL}, 61 \mathrm{mmol}, 1.2$ equiv) and sodium carbonate $\left(5.8 \mathrm{~g}, 55 \mathrm{mmol}, 1.1\right.$ equiv) at $0{ }^{\circ} \mathrm{C}$. The mixture was then stirred at room temperature for 6 hours. The resulting aqueous media was extracted with dichloromethane $(3 \times 100 \mathrm{~mL})$ and the combined organic extracts were dried over magnesium sulfate and concentrated on rotary evaporator. $3.86 \mathrm{~g}$ (55\% yield) of the spectroscopically pure triazine were obtained after recrystallization from a 9:1 isopropanol/heptane mixture as a brown solid. ${ }^{1} \mathrm{H}$ NMR (250 MHz, chloroform- $d$ ): $\delta_{\mathrm{H}} 8.81(\mathrm{~s}, 1 \mathrm{H}), 2.67$ (s, 3H), 2.49 (s, 3H). ${ }^{13} \mathrm{C}\left\{{ }^{1} \mathrm{H}\right\}$ NMR (63 MHz, chloroform- $d$ ): $\delta_{\mathrm{C}} 173.5(\mathrm{C}), 159.0(\mathrm{C}), 146.0(\mathrm{CH}), 21.8\left(\mathrm{CH}_{3}\right)$, $14.0\left(\mathrm{CH}_{3}\right)$. ESI ${ }^{+}-\mathrm{HRAM}: \mathrm{m} / \mathrm{z}$ calculated for $\left[\mathrm{C}_{5} \mathrm{H}_{8} \mathrm{~N}_{3} \mathrm{~S}\right]^{+}\left([\mathrm{M}+\mathrm{H}]^{+}\right)$142.043345, found 142.043308. IR (neat): $v_{\max } 3006,2928,1544,1422,1249 . \mathrm{mp}=72-73{ }^{\circ} \mathrm{C}$.

General procedure A for 3-vinyl-1,2,4-triazines (1) synthesis from boronic acid pinacol esters

3-methylthio-1,2,4-triazine, copper(I) 3-methylsalicylate (2.2 equiv) and vinylboronic acid pinacol ester (2.2 equiv) were dissolved in dry and degassed tetrahydrofuran $(0.1 \mathrm{M}$ of 
triazine) under argon atmosphere. The solution was degassed by argon bubbling for 15 minutes before tetrakis(triphenylphosphine)palladium ( 5 mol\%) was added. The mixture was subjected to argon bubbling for extra 15 minutes and subsequently heated up to reflux during 20 to 42 hours. Once cooled down to room temperature, the reaction mixture was filtered through a pad of diatomaceous earth. A $0.3 \mathrm{M}$ aqueous solution of sodium bicarbonate was then added to the filtrate and the mixture was extracted with dichloromethane (three times). The combined organic layers were dried over magnesium sulfate and concentrated on rotary evaporator. The expected substituted 3-vinyl-1,2,4-triazine was finally isolated after silica gel column chromatography.

\section{General procedure B for 3-vinyl-1,2,4-triazines (1) synthesis from boronic acids.}

3-methylthio-5-phenyl-1,2,4-triazine, copper(I) 3-methylsalicylate (2.2 equiv) and vinylboronic acid (2.2 equiv) were dissolved in a degassed 9:1 tetrahydrofuran/water mixture ( $0.1 \mathrm{M}$ of triazine) under argon atmosphere. The solution was degassed by argon bubbling for 15 minutes before tetrakis(triphenylphosphine)palladium (5 mol\%) was added. The mixture was subjected to argon bubbling for extra 15 minutes and subsequently heated up to reflux during 20 to 48 hours. Once cooled down to room temperature, the reaction mixture was filtered through a pad of diatomaceous earth. A $0.3 \mathrm{M}$ aqueous solution of sodium bicarbonate was then added to the filtrate and the mixture was extracted with dichloromethane (three times). The combined organic layers were dried over magnesium sulfate and concentrated on rotary evaporator. The attempted substituted 3-vinyl-1,2,4-triazine was finally isolated by silica gel column chromatography.

\section{(E)-3-(hept-1-en-1-yl)-5-phenyl-1,2,4-triazine (1e).}

Following the general procedure A from 3-methylthio-5-phenyl-1,2,4-triazine (500 mg, 2.46 $\mathrm{mmol})$ and hept-1-en-1-ylboronic acid pinacol ester $(1.22 \mathrm{~g}, 5.41 \mathrm{mmol}, 2.2 \text { equiv })^{30}$ for 24 hours, the title compound (429 mg, 69\% yield) was obtained as a yellow oil after purification 
by column chromatography (eluent $=9: 1$ petroleum ether/ethyl acetate, $\mathrm{R}_{f}=0.2$ ).

${ }^{1} \mathrm{H}$ NMR (400 MHz, chloroform- $d$ ): $\delta_{\mathrm{H}} 9.46(\mathrm{~s}, 1 \mathrm{H}), 8.23-8.16(\mathrm{~m}, 2 \mathrm{H}), 7.63-7.53(\mathrm{~m}, 3 \mathrm{H})$, $7.46(\mathrm{dt}, J=15.6,7.0 \mathrm{~Hz}, 1 \mathrm{H}), 6.82(\mathrm{dt}, J=15.7,1.5 \mathrm{~Hz}, 1 \mathrm{H}), 2.39(\mathrm{qd}, J=7.2,1.6 \mathrm{~Hz}, 2 \mathrm{H})$, $1.65-1.53(\mathrm{~m}, 2 \mathrm{H}), 1.43-1.34(\mathrm{~m}, 4 \mathrm{H}), 0.98-0.85(\mathrm{~m}, 3 \mathrm{H}) .{ }^{13} \mathrm{C}\left\{{ }^{1} \mathrm{H}\right\}$ NMR $(101 \mathrm{MHz}$, chloroform- $d$ ): $\delta_{\mathrm{C}} 163.7(\mathrm{C}), 155.0(\mathrm{C}), 144.9(\mathrm{CH}), 143.9(\mathrm{CH}), 134.0(\mathrm{C}), 132.4(\mathrm{CH}), 129.5$ $(2 \mathrm{CH}), 127.7(2 \mathrm{CH}), 127.0(\mathrm{CH}), 33.1\left(\mathrm{CH}_{2}\right), 31.6\left(\mathrm{CH}_{2}\right), 28.4\left(\mathrm{CH}_{2}\right), 22.7\left(\mathrm{CH}_{2}\right), 14.2$ $\left(\mathrm{CH}_{3}\right)$. ESI ${ }^{+}-\mathrm{HRAM}: \mathrm{m} / \mathrm{z}$ calculated for $\left[\mathrm{C}_{16} \mathrm{H}_{20} \mathrm{~N}_{3}\right]^{+}\left([\mathrm{M}+\mathrm{H}]^{+}\right)$254.165174, found 254.165147. IR (neat): $v_{\max } 2955,2927,2856,1538,1501,1366,765,689$.

\section{(E)-3-(2-cyclopropylvinyl)-5-phenyl-1,2,4-triazine (1f).}

Following the general procedure A from 3-methylthio-5-phenyl-1,2,4-triazine (456 mg, 2.25 $\mathrm{mmol})$ and 2-cyclopropylvinylboronic acid pinacol ester (1.00 g, $4.95 \mathrm{mmol}, 2.2$ equiv) for 24 hours, the title compound (315 mg, 63\% yield) was obtained as yellow solid after purification by silica gel column chromatography (eluent $=8: 2$ petroleum ether/ethyl acetate, $\mathrm{R}_{f}=0.4$ ).

${ }^{1} \mathrm{H}$ NMR (400 MHz, chloroform- $d$ ): $\delta_{\mathrm{H}} 9.42(\mathrm{~s}, 1 \mathrm{H}), 8.22-8.12(\mathrm{~m}, 2 \mathrm{H}), 7.63-7.50(\mathrm{~m}, 3 \mathrm{H})$, $6.99-6.83(\mathrm{~m}, 2 \mathrm{H}), 1.85-1.71(\mathrm{~m}, 1 \mathrm{H}), 1.06-0.94(\mathrm{~m}, 2 \mathrm{H}), 0.79-0.68(\mathrm{~m}, 2 \mathrm{H}) .{ }^{13} \mathrm{C}\left\{{ }^{1} \mathrm{H}\right\}$ NMR (101 MHz, chloroform- $d$ ): $\delta_{\mathrm{C}} 163.6$ (C), $154.9(\mathrm{C}), 149.1(\mathrm{CH}), 143.6(\mathrm{C}), 134.0(\mathrm{C})$, $132.3(\mathrm{CH}), 129.4(2 \mathrm{CH}), 127.6(2 \mathrm{CH}), 124.2(\mathrm{C}), 15.4(\mathrm{CH}), 8.8\left(2 \mathrm{CH}_{2}\right) . \mathrm{ESI}^{+}$-HRAM: $\mathrm{m} / \mathrm{z}$ calculated for $\left[\mathrm{C}_{14} \mathrm{H}_{14} \mathrm{~N}_{3}\right]^{+}\left([\mathrm{M}+\mathrm{H}]^{+}\right)$224.118224, found 224.117939. IR (neat): $v_{\max }$ $3057,3010,2926,1645,1537,1508,1283,944,761,687 . \mathrm{mp}=65{ }^{\circ} \mathrm{C}$.

\section{(E)-5-phenyl-3-(3-phenylprop-1-en-1-yl)-1,2,4-triazine (1g)}

Following the general procedure B from 3-methylthio-5-phenyl-1,2,4-triazine (553 mg, 2.72 mmol) and (3-phenylprop-1-en-1-yl)boronic acid (1.00 g, $5.99 \mathrm{mmol}, 2.2$ equiv) for 36 hours, the title compound (119 mg, 16\% yield) was obtained as a yellow solid after purification by silica gel column chromatography (eluent $=9: 1$ pentane/ethyl acetate, $\mathbf{R}_{f}=0.4$ ).

${ }^{1} \mathrm{H}$ NMR (400 MHz, chloroform- $d$ ): $\delta_{\mathrm{H}} 9.48(\mathrm{~s}, 1 \mathrm{H}), 8.23-8.08(\mathrm{~m}, 2 \mathrm{H}), 7.68-7.44(\mathrm{~m}, 4 \mathrm{H})$, 
$7.38-7.23(\mathrm{~m}, 5 \mathrm{H}), 6.86(\mathrm{dt}, J=15.5,1.6 \mathrm{~Hz}, 1 \mathrm{H}), 3.73(\mathrm{dd}, J=7.0,1.6 \mathrm{~Hz}, 2 \mathrm{H}) .{ }^{13} \mathrm{C}\left\{{ }^{1} \mathrm{H}\right\}$ NMR (101 MHz, chloroform- $d$ ): $\delta_{\mathrm{C}} 163.6(\mathrm{C}), 155.0(\mathrm{C}), 144.0(\mathrm{CH}), 142.6(\mathrm{CH}), 138.7(\mathrm{C})$, $133.9(\mathrm{C}), 132.5(\mathrm{CH}), 129.5(2 \mathrm{CH}), 129.1(2 \mathrm{CH}), 128.8(2 \mathrm{CH}), 128.1(\mathrm{CH}), 127.7(2 \mathrm{CH})$, $126.7(\mathrm{CH}), 39.4\left(\mathrm{CH}_{2}\right)$. ESI ${ }^{+}-\mathrm{HRAM}: \mathrm{m} / \mathrm{z}$ calculated for $\left[\mathrm{C}_{18} \mathrm{H}_{16} \mathrm{~N}_{3}\right]^{+}\left([\mathrm{M}+\mathrm{H}]^{+}\right) 274.133874$, found 274.133688. IR (neat): $v_{\max } 3053,2911,1539,1508,1316,974,693 . \mathrm{mp}=63-64{ }^{\circ} \mathrm{C}$.

\section{(E)-3-(2-cyclohexylvinyl)-5-phenyl-1,2,4-triazine (1h)}

Following the general procedure A from 3-methylthio-5-phenyl-1,2,4-triazine (781 mg, 3.84 $\mathrm{mmol})$ and (2-cyclohexylvinyl)boronic acid pinacol ester $(2.00 \mathrm{~g}, 8.45 \mathrm{mmol}, 2.2 \text { equiv })^{30}$ for 24 hours, the title compound (164 mg, 16\% yield) was obtained as an orange oil after purification by silica gel column chromatography (eluent $=$ dichloromethane, $\mathbf{R}_{f}=0.2$ ).

${ }^{1} \mathrm{H}$ NMR (400 MHz, chloroform- $d$ ): $\delta_{\mathrm{H}} 9.46(\mathrm{~s}, 1 \mathrm{H}), 8.26-8.04(\mathrm{~m}, 2 \mathrm{H}), 7.61-7.52(\mathrm{~m}, 3 \mathrm{H})$, $7.41(\mathrm{dd}, J=16.0,6.9 \mathrm{~Hz}, 1 \mathrm{H}), 6.77(\mathrm{dd}, J=16.0,1.5 \mathrm{~Hz}, 1 \mathrm{H}), 2.39-2.26(\mathrm{~m}, 1 \mathrm{H}), 1.95-$ $1.86(\mathrm{~m}, 2 \mathrm{H}), 1.86-1.77(\mathrm{~m}, 2 \mathrm{H}), 1.76-1.68(\mathrm{~m}, 1 \mathrm{H}), 1.40-1.20(\mathrm{~m}, 5 \mathrm{H}) .{ }^{13} \mathrm{C}\left\{{ }^{1} \mathrm{H}\right\} \mathrm{NMR}$ (101 MHz, chloroform- $d$ ): $\delta_{\mathrm{C}} 164.0(\mathrm{C}), 155.1(\mathrm{C}), 149.8(\mathrm{CH}), 143.8(\mathrm{CH}), 134.0(\mathrm{C}), 132.4$ (CH), $129.4(2 \mathrm{CH}), 127.7(2 \mathrm{CH}), 124.7(\mathrm{CH}), 41.3(\mathrm{CH}), 32.3\left(2 \mathrm{CH}_{2}\right), 26.2\left(\mathrm{CH}_{2}\right), 26.1$ (2 $\left.\mathrm{CH}_{2}\right)$. ESI ${ }^{+}-\mathrm{HRAM}: \mathrm{m} / \mathrm{z}$ calculated for $\left[\mathrm{C}_{17} \mathrm{H}_{20} \mathrm{~N}_{3}\right]^{+}\left([\mathrm{M}+\mathrm{H}]^{+}\right)$266.165174, found 266.165352. IR (neat): $\nu_{\max } 2923,2850,1537,1503,1360,1282,977,689$.

\section{(E)-5-phenyl-3-styryl-1,2,4-triazine (1i).}

Following the general procedure B from 3-methylthio-5-phenyl-1,2,4-triazine (203 mg, 1.00 mmol) and styrylboronic acid (326 mg, $2.2 \mathrm{mmol}, 2.2$ equiv) for 48 hours, the title compound (71 mg, 27\% yield) was obtained as a yellow oil after purification by silica gel column chromatography (eluent $=9: 1$ petroleum ether/ethyl acetate, $R_{f}=0.2$ ).

${ }^{1} \mathrm{H}$ NMR $\left(250 \mathrm{MHz}\right.$, chloroform- $d$ ): $\delta_{\mathrm{H}} 9.50(\mathrm{~s}, 1 \mathrm{H}), 8.29-8.19(\mathrm{~m}, 3 \mathrm{H}), 7.73-7.67(\mathrm{~m}, 2 \mathrm{H})$, $7.65-7.56(\mathrm{~m}, 3 \mathrm{H}), 7.49(\mathrm{~d}, J=16.1 \mathrm{~Hz}, 1 \mathrm{H}), 7.49-7.37(\mathrm{~m}, 3 \mathrm{H}) .{ }^{13} \mathrm{C}\left\{{ }^{1} \mathrm{H}\right\} \mathrm{NMR}(63 \mathrm{MHz}$, chloroform- $d$ ): $\delta_{\mathrm{C}} 164.1(\mathrm{C}), 155.1(\mathrm{C}), 143.9(\mathrm{CH}), 140.1(\mathrm{CH}), 135.8(\mathrm{C}), 134.0(\mathrm{C}), 132.5$ 
$(\mathrm{CH}), 129.8(\mathrm{CH}), 129.5(2 \mathrm{CH}), 129.1(2 \mathrm{CH}), 128.1(2 \mathrm{CH}), 127.8(2 \mathrm{CH}), 124.7(\mathrm{CH})$. ESI ${ }^{+}$-HRAM: $\mathrm{m} / \mathrm{z}$ calculated for $\left[\mathrm{C}_{17} \mathrm{H}_{14} \mathrm{~N}_{3}\right]^{+}\left([\mathrm{M}+\mathrm{H}]^{+}\right)$260.118224, found 260.118214. IR (neat): $v_{\max } 3056,1636,1537,1505,1451,973,767,681$.

\section{(E)-5-methyl-3-(prop-1-en-1-yl)-1,2,4-triazine (1j)}

Following the general procedure A from 5-methyl-3-methylthio-1,2,4-triazine (370 mg, 2.62 mmol) and trans-1-propenylboronic acid pinacol ester (1.00 g, $5.77 \mathrm{mmol}, 2.2$ equiv) for 24 hours, the title compound (57 mg, 16\% yield) was obtained as a light-yellow solid after purification by silica gel column chromatography (eluent $=$ gradient from dichloromethane to 9:1 dichloromethane/ethyl acetate, $\left.\mathrm{R}_{f}\left(9: 1 \mathrm{CH}_{2} \mathrm{Cl}_{2} / \mathrm{EtOAc}\right)=0.4\right)$.

${ }^{1} \mathrm{H}$ NMR (400 MHz, chloroform- $d$ ): $\delta_{\mathrm{H}} 8.89(\mathrm{~s}, 1 \mathrm{H}), 7.31(\mathrm{dq}, J=15.6,6.9 \mathrm{~Hz}, 1 \mathrm{H}), 6.72(\mathrm{dq}$, $J=15.6,1.7 \mathrm{~Hz}, 1 \mathrm{H}), 2.52(\mathrm{~s}, 3 \mathrm{H}), 2.01(\mathrm{dd}, J=6.9,1.7 \mathrm{~Hz}, 3 \mathrm{H}) .{ }^{13} \mathrm{C}\left\{{ }^{1} \mathrm{H}\right\} \mathrm{NMR}(101 \mathrm{MHz}$, chloroform-d): $\delta_{\mathrm{C}} 163.3(\mathrm{C}), 159.0(\mathrm{C}), 147.7(\mathrm{CH}), 139.4(\mathrm{CH}), 128.2(\mathrm{CH}), 22.0\left(\mathrm{CH}_{3}\right)$, $18.7\left(\mathrm{CH}_{3}\right)$. ESI ${ }^{+}-\mathrm{HRAM}: \mathrm{m} / \mathrm{z}$ calculated for $\left[\mathrm{C}_{7} \mathrm{H}_{10} \mathrm{~N}_{3}\right]^{+}\left([\mathrm{M}+\mathrm{H}]^{+}\right)$136.086924, found 136.086793. IR (neat): $v_{\max } 3043,2917,1655,1548,1356,1283,965 . \mathrm{mp}=77-79{ }^{\circ} \mathrm{C}$.

\section{(E)-3-(prop-1-en-1-yl)-5-(trifluoromethyl)-1,2,4-triazine (1k).}

Following the general procedure A from 3-methylthio-5-trifluoromethyl-1,2,4-triazine (512 $\mathrm{mg}, 2.62 \mathrm{mmol})$ and trans-1-propenylboronic acid pinacol ester (1.00 g, $5.77 \mathrm{mmol}, 2.2$ equiv) for 24 hours, the title compound (349 mg, 67\% yield) was obtained as volatile brightyellow liquid after purification by column chromatography (dry loading, eluent $=$ gradient from pentane to $95: 5$ pentane/diethyl ether, $\mathrm{R}_{f}(95: 5$ petr. eth./Et $\left.2 \mathrm{O})=0.5\right)$. N.B.: the solution obtained after column chromatography should not be heated on rotary evaporator for concentration (volatile compound).

${ }^{1} \mathrm{H}$ NMR (400 MHz, chloroform-d): $\delta_{\mathrm{H}} 9.37$ (s, 1H), 7.52 (dq, $\left.J=15.6,7.0 \mathrm{~Hz}, 1 \mathrm{H}\right), 6.89$ (dq, $J=15.6,1.8 \mathrm{~Hz}, 1 \mathrm{H}), 2.09(\mathrm{dd}, J=7.0,1.8 \mathrm{~Hz}, 3 \mathrm{H}) .{ }^{13} \mathrm{C}\left\{{ }^{1} \mathrm{H}\right\} \mathrm{NMR}(63 \mathrm{MHz}$, chloroform- $d)$ : $\delta_{\mathrm{C}} 163.9(\mathrm{C}), 147.3\left(\mathrm{q},{ }^{2} J_{\mathrm{C}-\mathrm{F}}=37.6 \mathrm{~Hz}, \mathrm{C}\right), 143.1(\mathrm{CH}), 142.1\left(\mathrm{q},{ }^{3} J_{\mathrm{C}-\mathrm{F}}=2.5 \mathrm{~Hz}, \mathrm{CH}\right), 126.8$ 
$(\mathrm{CH}), 120.1\left(\mathrm{q},{ }^{1} J_{\mathrm{C}-\mathrm{F}}=276.2 \mathrm{~Hz}, \mathrm{CF}_{3}\right), 18.8\left(\mathrm{CH}_{3}\right) . \mathrm{ESI}^{+}-\mathrm{HRAM}: \mathrm{m} / \mathrm{z}$ calculated for $\left[\mathrm{C}_{7} \mathrm{H}_{7} \mathrm{~F}_{3} \mathrm{~N}_{3}\right]^{+}\left([\mathrm{M}+\mathrm{H}]^{+}\right)$190.058658, found 190.058495. IR (neat): $v_{\max }$ 3050, 2973, 2858, $1418,1199,1144$.

\section{(E)-5,6-dimethyl-3-(prop-1-en-1-yl)-1,2,4-triazine (11).}

Following the general procedure A from 5,6-dimethyl-3-methylthio-1,2,4-triazine (407 mg, $2.62 \mathrm{mmol})$ and trans-1-propenylboronic acid pinacol ester (1.00 g, $5.77 \mathrm{mmol}, 2.2$ equiv) for 20 hours, the title compound (373 mg, 95\% yield) was obtained as a beige solid after purification by silica gel column chromatography (eluent $=8: 2$ pentane/ethyl acetate, $\mathrm{R}_{f}=$ $0.3)$.

${ }^{1} \mathrm{H}$ NMR $\left(250 \mathrm{MHz}\right.$, chloroform- $d$ ): $\delta_{\mathrm{H}} 7.23(\mathrm{dq}, J=15.6,7.0 \mathrm{~Hz}, 1 \mathrm{H}), 6.69(\mathrm{dq}, J=15.6,1.7$ $\mathrm{Hz}, 1 \mathrm{H}), 2.63(\mathrm{~s}, 3 \mathrm{H}), 2.49(\mathrm{~s}, 3 \mathrm{H}), 1.99(\mathrm{dd}, J=7.0,1.7 \mathrm{~Hz}, 3 \mathrm{H}) .{ }^{13} \mathrm{C}\left\{{ }^{1} \mathrm{H}\right\} \mathrm{NMR}(63 \mathrm{MHz}$, chloroform- $d)$ : $\delta_{\mathrm{C}} 162.2(\mathrm{C}), 158.3(\mathrm{C}), 155.1(\mathrm{C}), 137.8(\mathrm{CH}), 128.2(\mathrm{CH}), 22.0\left(\mathrm{CH}_{3}\right), 19.6$ $\left(\mathrm{CH}_{3}\right), 18.6\left(\mathrm{CH}_{3}\right)$. ESI ${ }^{+}-\mathrm{HRAM}: \mathrm{m} / \mathrm{z}$ calculated for $\left[\mathrm{C}_{8} \mathrm{H}_{12} \mathrm{~N}_{3}\right]^{+}\left([\mathrm{M}+\mathrm{H}]^{+}\right) 150.102574$, found 150.102505. IR (neat): $v_{\max } 3012,2935,2858,1653,1541,1517,995 . \mathrm{mp}=41-42{ }^{\circ} \mathrm{C}$.

\section{(E)-3-(prop-1-en-1-yl)-1,2,4-triazine (1m).}

Following the general procedure A from 3-methylthio-1,2,4-triazine (333 mg, $2.62 \mathrm{mmol})$ and trans-1-propenylboronic acid pinacol ester $(1.00 \mathrm{~g}, 5.77 \mathrm{mmol}, 2.2$ equiv) for 20 hours, the title compound (172 mg, 54\% yield) was obtained as yellow crystals after purification by silica gel column chromatography (eluent $=8: 2$ pentane/ethyl acetate, $\mathrm{R}_{f}=0.4$ ).

${ }^{1} \mathrm{H}$ NMR (250 MHz, chloroform- $d$ ): $\delta_{\mathrm{H}} 9.01(\mathrm{~d}, J=2.4 \mathrm{~Hz}, 1 \mathrm{H}), 8.50(\mathrm{~d}, J=2.4 \mathrm{~Hz}, 1 \mathrm{H}), 7.34$ $(\mathrm{dq}, J=15.6,6.9 \mathrm{~Hz}, 1 \mathrm{H}), 6.77(\mathrm{dq}, J=15.6,1.7 \mathrm{~Hz}, 1 \mathrm{H}), 2.03(\mathrm{dd}, J=6.9,1.7 \mathrm{~Hz}, 3 \mathrm{H})$. ${ }^{13} \mathrm{C}\left\{{ }^{1} \mathrm{H}\right\}$ NMR (63 MHz, chloroform- $\left.d\right): \delta_{\mathrm{C}} 164.4(\mathrm{C}), 148.6(\mathrm{CH}), 147.2(\mathrm{CH}), 140.1(\mathrm{CH})$, $128.1(\mathrm{CH}), 18.8\left(\mathrm{CH}_{3}\right) . \mathrm{ESI}^{+}-\mathrm{HRAM}: \mathrm{m} / \mathrm{z}$ calculated for $\left[\mathrm{C}_{6} \mathrm{H}_{8} \mathrm{~N}_{3}\right]^{+}\left([\mathrm{M}+\mathrm{H}]^{+}\right) 122.071274$, found 122.071296. IR (neat): $v_{\max } 3050,3034,2966,2943,2915,1655,1550,1527,1432$, 1409, 1553. $\mathrm{mp}=67^{\circ} \mathrm{C}$. 


\section{$N$-methoxypropargylamine (2g).}

$240 \mathrm{~mL}$ of a $5 \mathrm{M}$ hydrochloric acid solution (1.2 mol, 10 equiv) in a water/methanol mixture, obtained by mixing $100 \mathrm{~mL}$ of a $37 \%$ aqueous hydrochloric acid solution and $140 \mathrm{~mL}$ of methanol, were added to stirred $N$-(tert-butoxycarbonyl)- $N$-(propargyl)methoxyamine $(21.5 \mathrm{~g}$, $116 \mathrm{mmol}$, prepared according to literature procedure). ${ }^{31}$ The mixture was subsequently refluxed for 3 hours (TLC monitoring using ethyl acetate as eluent). Once cooled down to room temperature, reactional mixture was diluted with $150 \mathrm{~mL}$ of distilled water and $150 \mathrm{~mL}$ of dichloromethane. Layers were separated, aqueous layer was washed with dichloromethane $(150 \mathrm{~mL})$ and ethyl acetate $(150 \mathrm{~mL})$ and finally evaporated on rotatory evaporator and dried under vacuum. The title ammonium salt was obtained as a white crystalline solid $(8.71 \mathrm{~g}, 61 \%$ yield).

$\mathrm{N}$-methoxypropargylamine hydrochloride $(4.95 \mathrm{~g}, 40.7 \mathrm{mmol})$ was introduced in a $250 \mathrm{~mL}$ round bottom flask with $125 \mathrm{~mL}$ of diethyl ether. $90 \mathrm{~mL}$ (2.2 equiv) of a $1 \mathrm{M}$ sodium hydroxide aqueous solution were then added and the biphasic mixture was vigorously stirred for 15 minutes. Layers were separated, aqueous phase was extracted with diethyl ether $(2 \times 50$ $\mathrm{mL})$. Gathered organic extracts were dried over magnesium sulfate and concentrated on rotary evaporator in a $0-5 \quad{ }^{\circ} \mathrm{C}$ cold bath. $3.28 \mathrm{~g} \quad(95 \%$ yield $)$ of the expected $N$-methoxypropargylamine were obtained as a colorless liquid.

${ }^{1} \mathrm{H}$ NMR $(250 \mathrm{MHz}$, chloroform- $d): \delta_{\mathrm{H}} 5.56(\mathrm{t}, J=6.8 \mathrm{~Hz}, 1 \mathrm{H}), 3.67(\mathrm{dd}, J=6.8,2.5 \mathrm{~Hz}, 2 \mathrm{H})$, $3.58(\mathrm{~s}, 3 \mathrm{H}), 2.25(\mathrm{t}, J=2.5 \mathrm{~Hz}, 1 \mathrm{H}) .{ }^{13} \mathrm{C} \mathrm{NMR}\left(63 \mathrm{MHz}\right.$, chloroform- $d$ ): $\delta_{\mathrm{C}} 80.3(\mathrm{C}), 71.9$ $(\mathrm{CH}), 62.1\left(\mathrm{CH}_{3}\right), 41.1\left(\mathrm{CH}_{2}\right)$. ESI ${ }^{+}-\mathrm{HRAM}$ : the compound is too volatile to be detected.

\section{$O$-benzyl- $N$-(prop-2-yn-1-yl)hydroxylamine (2h).}

A $5 \mathrm{M}$ hydrochloric acid solution in a water/methanol mixture (obtained by diluting $3.2 \mathrm{~mL}$ of $37 \%$ aqueous hydrochloric acid in $4.4 \mathrm{~mL}$ of methanol, $7.6 \mathrm{~mL}, 38 \mathrm{mmol}, 10$ equiv) was slowly added to tert-butyl (benzyloxy)(prop-2-yn-1-yl)carbamate (1.00 g, $3.83 \mathrm{mmol}$, 
prepared according to literature procedure $)^{31}$ and the mixture was stirred 24 hours at room temperature (TLC monitoring, eluent $=9: 1$ petroleum ether/EtOAc, $\mathrm{R}_{f}=0.5$ ). $15 \mathrm{~mL}$ of dichloromethane were then added into the flask. After 15 minutes of stirring, phases were separated, and aqueous layer was washed with $25 \mathrm{~mL}$ of dichloromethane. $25 \mathrm{~mL}$ of sodium bicarbonate saturated aqueous solution were added to the aqueous phase which was subsequently extracted with dichloromethane $(2 \times 25 \mathrm{~mL})$. The combined organic extracts were dried over magnesium sulfate and concentrated on rotary evaporator at room temperature, affording the spectroscopically pure propargylamine $\mathbf{2 h}$ (455 $\mathrm{mg}, 74 \%$ yield).

${ }^{1} \mathrm{H}$ NMR (250 MHz, chloroform- $d$ ): $\delta_{\mathrm{H}} 7.44-7.25$ (m, 5H), 5.52 (br s, 1H), 4.77 (s, 2H), 3.66 (s), $2.25(\mathrm{t}, \mathrm{J}=2.5 \mathrm{~Hz}, 1 \mathrm{H}) .{ }^{13} \mathrm{C}\left\{{ }^{1} \mathrm{H}\right\}$ NMR $(63 \mathrm{MHz}$, chloroform- $d): \delta_{\mathrm{C}} 137.8(\mathrm{C}), 128.6(2$ $\mathrm{CH}), 128.61(2 \mathrm{CH}), 128.55(\mathrm{CH}), 80.3(\mathrm{C}), 76.5\left(\mathrm{CH}_{2}\right), 72.0(\mathrm{CH}), 41.5\left(\mathrm{CH}_{2}\right) . \mathrm{ESI}^{+}-\mathrm{HRAM}$ $\mathrm{m} / \mathrm{z}$ calculated for $\left[\mathrm{C}_{10} \mathrm{H}_{12} \mathrm{NO}\right]^{+}\left([\mathrm{M}+\mathrm{H}]^{+}\right)$162.091340, found 162.091115. IR (neat): $v_{\max }$ 3288, 2915, 2859, 1545, 697, 636.

General procedure $C$ for the aza-Michael reaction to 3-vinyl-1,2,4-triazine. The alkoxyamine derivative 2 (1.5 equiv) was added to a solution of 3-vinyl-1,2,4-triazines 1 (0.1$1 \mathrm{mmol}, 0.1 \mathrm{M})$ and trifluoromethanesulfonimide $\mathbf{E}(20 \mathrm{~mol} \%)$ in toluene. The mixture was stirred at room temperature until a complete conversion of starting material $\mathbf{1}$ was observed by TLC. A saturated aqueous solution of sodium bicarbonate was then added and the organic phase was separated. The aqueous layer was extracted by dichloromethane (twice). The combined organic layers were dried over anhydrous magnesium sulfate and concentrated on rotary evaporator. The crude product was purified by silica gel column chromatography to yield the desired adduct 3 . If required the yield was also estimated on the crude product by ${ }^{1} \mathrm{H}$ NMR thanks to dibenzylether (0.25 equiv.) as an internal standard.

$N$-benzyl- $N$-methyl-2-(5-phenyl-1,2,4-triazin-3-yl)ethan-1-amine (3a).

$N$-methybenzylamine (97\%, $20 \mu \mathrm{L}, 18.8 \mathrm{mg}, 0.15 \mathrm{mmol}, 1.5$ equiv) was added into a solution 
of 5 phenyl-3-vinyl-1,2,4-triazine $(18.3 \mathrm{mg}, 0.10 \mathrm{mmol})$ in $1 \mathrm{~mL}$ of methanol. The mixture was stirred at $30{ }^{\circ} \mathrm{C}$ for 24 hours, concentrated on rotary evaporator and purified by column chromatography (eluent $=1: 4$ petroleum ether/ethyl acetate, $\mathrm{R}_{f}=0.3$ ) to afford the tittle product (31 mg, $0.10 \mathrm{mmol}$, quantitative yield) as a yellow oil.

${ }^{1} \mathrm{H}$ NMR (400 MHz, chloroform- $d$ ): $\delta_{\mathrm{H}} 9.55(\mathrm{~s}, 1 \mathrm{H}), 8.17-8.10(\mathrm{~m}, 2 \mathrm{H}), 7.63-7.51(\mathrm{~m}, 3 \mathrm{H})$, $7.24-7.15(\mathrm{~m}, 5 \mathrm{H}), 3.59(\mathrm{~s}, 2 \mathrm{H}), 3.40(\mathrm{t}, J=7.2 \mathrm{~Hz}, 2 \mathrm{H}), 3.06(\mathrm{t}, J=7.2 \mathrm{~Hz}, 2 \mathrm{H}), 2.32(\mathrm{~s}$, 3H). ${ }^{13} \mathrm{C}\left\{{ }^{1} \mathrm{H}\right\}$ NMR (100.7 MHz, chloroform- $\left.d\right) \delta_{\mathrm{C}} 169.0(\mathrm{C}), 155.2(\mathrm{C}), 144.3(\mathrm{CH}), 139.1$ (C), $133.8(\mathrm{C}), 132.5(\mathrm{CH}), 129.5(2 \mathrm{CH}), 129.1(2 \mathrm{CH}), 128.3(2 \mathrm{CH}), 127.8(2 \mathrm{CH}), 127.0$ (CH), $62.2\left(\mathrm{CH}_{2}\right), 55.7(\mathrm{CH} 2), 42.2\left(\mathrm{CH}_{3}\right), 35.5\left(\mathrm{CH}_{2}\right)$. ESI ${ }^{+}-\mathrm{HRAM}: \mathrm{m} / \mathrm{z}$ calculated for $\left[\mathrm{C}_{19} \mathrm{H}_{21} \mathrm{~N}_{4}\right]^{+}\left([\mathrm{M}+\mathrm{H}]^{+}\right)$305.176073, found 305.176150. IR (neat): $v_{\max } 3058,3027,2788$, $1601,1544,1508,1495,1445,1319,1075,1046,738,690$.

$N$-Benzyl- $O$-methyl- $N$-(1-(5-phenyl-1,2,4-triazin-3-yl)propan-2-yl)hydroxylamine $\quad(3 \mathrm{~b})$. Following the general procedure C from 5-phenyl-3-(prop-1-en-1-yl)-1,2,4-triazine 1 b (19.7 $\mathrm{mg}, 0.10 \mathrm{mmol})$ and $N$-methoxybenzylamine $2 \mathrm{~d}(19.8 \mu \mathrm{L}, 0.15 \mathrm{mmol}, 1.5$ equiv) in 24 hours, the title compound (32.4 mg, 97\% yield) was obtained as a yellow oil after purification by column chromatography (9:1 pentane/AcOEt, $\left.R_{f}=0.3\right) .{ }^{1} \mathrm{H}$ NMR $(400 \mathrm{MHz}$, chloroform- $d)$ : $\delta_{\mathrm{H}} 9.55(\mathrm{~s}, 1 \mathrm{H}), 8.16-8.08(\mathrm{~m}, 2 \mathrm{H}), 7.64-7.51(\mathrm{~m}, 3 \mathrm{H}), 7.26-7.16(\mathrm{~m}, 5 \mathrm{H}), 3.98(\mathrm{~d}, J=13.0$ $\mathrm{Hz}, 1 \mathrm{H}), 3.83(\mathrm{~d}, J=13.0 \mathrm{~Hz}, 1 \mathrm{H}), 3.76(\mathrm{~h}, J=6.8 \mathrm{~Hz}, 1 \mathrm{H}), 3.61(\mathrm{dd}, J=6.8,13.8 \mathrm{~Hz}, 1 \mathrm{H})$, $3.28(\mathrm{~s}, 3 \mathrm{H}), 3.22(\mathrm{dd}, J=6.8,13.8 \mathrm{~Hz}, 1 \mathrm{H}), 1.27(\mathrm{~d}, J=6.8 \mathrm{~Hz}, 3 \mathrm{H}) .{ }^{13} \mathrm{C}\left\{{ }^{1} \mathrm{H}\right\} \mathrm{NMR}(100.7$ MHz, chloroform- $d): \delta_{\mathrm{C}} 169.0(\mathrm{C}), 155.0(\mathrm{C}), 144.1(\mathrm{CH}), 138.2(\mathrm{C}), 133.8(\mathrm{C}), 132.5(\mathrm{CH})$, $129.6(2 \mathrm{CH}), 129.5(2 \mathrm{CH}), 128.2(2 \mathrm{CH}), 127.7(2 \mathrm{CH}), 127.2(\mathrm{CH}), 61.7\left(\mathrm{CH}_{3}\right), 59.3(\mathrm{CH})$, $58.0\left(\mathrm{CH}_{2}\right), 41.5\left(\mathrm{CH}_{2}\right), 14.6\left(\mathrm{CH}_{3}\right)$. ESI'-HRAM: $m / z$ calculated for $\left[\mathrm{C}_{20} \mathrm{H}_{23} \mathrm{~N}_{4} \mathrm{O}\right]^{+}\left([\mathrm{M}+\mathrm{H}]^{+}\right)$ 335.186638, found 335.186339. IR (neat): $v_{\max } 2968,2929,2853,1729,1544,1508,1445$, $1317,1049,762,732,691$. 
Following the general procedure A from 5-phenyl-3-(prop-1-en-2-yl)-1,2,4-triazine 1c (19.7 g, $0.10 \mathrm{mmol})$ and $N$-methoxybenzylamine $2 d(19.8 \mu \mathrm{L}, 0.15 \mathrm{mmol}, 1.5$ equiv) in 24 hours, the title compound $(25.9 \mathrm{~g}, 77 \%$ yield $)$ was obtained as a yellow oil after purification by column chromatography (eluent $=$ pentane/EtOAc, $\left.R_{f}=0.3\right) .{ }^{1} \mathrm{H}$ NMR $(400 \mathrm{MHz}$, chloroform- $d)$ : $\delta_{\mathrm{H}} 9.55(\mathrm{~s}, 1 \mathrm{H}), 8.14-8.01(\mathrm{~m}, 2 \mathrm{H}), 7.64-7.48(\mathrm{~m}, 3 \mathrm{H}), 7.19-7.14(\mathrm{~m}, 5 \mathrm{H})$, $3.93(\mathrm{~d}, J=12.8 \mathrm{~Hz}, 1 \mathrm{H}), 3.84-3.75(\mathrm{~m}, 1 \mathrm{H}), 3.77(\mathrm{~d}, 12.8 \mathrm{~Hz}, 1 \mathrm{H}), 3.37(\mathrm{dd}, J=12.9,9.5$ $\mathrm{Hz}, 1 \mathrm{H}), 3.33(\mathrm{~s}, 3 \mathrm{H}), 2.93(\mathrm{dd}, J=12.7,5.1 \mathrm{~Hz}, 1 \mathrm{H}), 1.41(\mathrm{~d}, J=7.0 \mathrm{~Hz}, 3 \mathrm{H}) .{ }^{13} \mathrm{C}\left\{{ }^{1} \mathrm{H}\right\} \mathrm{NMR}$ (101 MHz, chloroform- $d$ ): $\delta_{\mathrm{C}} 172.7(\mathrm{C}), 155.2(\mathrm{C}), 144.3(\mathrm{CH}), 137.4(\mathrm{C}), 134.0(\mathrm{C}), 132.4$ $(\mathrm{CH}), 129.5(2 \mathrm{CH}), 129.4(2 \mathrm{CH}) 128.2(2 \mathrm{CH}), 127.7(2 \mathrm{CH}), 127.3(\mathrm{CH}), 63.3\left(\mathrm{CH}_{2}\right), 62.9$ $\left(\mathrm{CH}_{2}\right), 61.0\left(\mathrm{CH}_{3}\right), 40.0(\mathrm{CH}), 18.6\left(\mathrm{CH}_{3}\right)$. ESI ${ }^{+}-\mathrm{HRAM}: \mathrm{m} / z$ calculated for $\left[\mathrm{C}_{20} \mathrm{H}_{23} \mathrm{~N}_{4} \mathrm{O}\right]^{+}$ $\left([\mathrm{M}+\mathrm{H}]^{+}\right)$335.186638, found 335.186299. IR (neat): $v_{\max } 3060,2971,2934,1542,1508$, 1319, 1049, 769, 692.

2-(benzyl(methoxy)amino)-3-(5-phenyl-1,2,4-triazin-3-yl)propan-1-ol (3d). Following the general procedure $\mathrm{C}$ from 3-(5-phenyl-1,2,4-triazin-3-yl)prop-2-en-1-ol 1d (21.3 mg, 0.10 mmol) and $N$-methoxybenzylamine $2 \mathbf{d}(19.8 \mu \mathrm{L}, 0.15 \mathrm{mmol}, 1.5$ equiv) in 24 hours, the title compound (29.8 $\mathrm{mg}, 85 \%$ yield) was obtained as a dark-yellow oil after purification by column chromatography (eluent $=1: 1$ pentane/EtOAc, $\left.R_{f}=0.4\right) .{ }^{1} \mathrm{H}$ NMR $(400 \mathrm{MHz}$, chloroform- $d): \delta_{\mathrm{H}} 9.56(\mathrm{~s}, 1 \mathrm{H}), 8.16-8.07(\mathrm{~m}, 2 \mathrm{H}), 7.65-7.51(\mathrm{~m}, 3 \mathrm{H}), 7.32-7.20(\mathrm{~m}, 5 \mathrm{H})$, $4.11(\mathrm{~d}, J=13 \mathrm{~Hz}, 1 \mathrm{H}), 4.02(\mathrm{~d}, J=13 \mathrm{~Hz}, 1 \mathrm{H}), 3.91-3.78(\mathrm{~m}, 2 \mathrm{H}), 3.71(\mathrm{dd}, J=14,5.5 \mathrm{~Hz}$, 1H), $3.68-3.60(\mathrm{~m}, 1 \mathrm{H}), 3.37(\mathrm{~s}, 3 \mathrm{H}), 3.29(\mathrm{dd}, J=14,7 \mathrm{~Hz}, 1 \mathrm{H}), 2.91$ (br s, $1 \mathrm{H}) .{ }^{13} \mathrm{C}\left\{{ }^{1} \mathrm{H}\right\}$ NMR (101 MHz, chloroform-d): $\delta \mathrm{C} 168.6(\mathrm{C}), 155.2(\mathrm{C}), 144.2(\mathrm{CH}), 137.2(\mathrm{C}), 133.5(\mathrm{C})$, $132.7(\mathrm{CH}), 129.7(2 \mathrm{CH}), 129.5(2 \mathrm{CH}), 128.4(2 \mathrm{CH}), 127.7(2 \mathrm{CH}), 127.6(\mathrm{CH}), 64.6(\mathrm{CH})$, $62.3\left(\mathrm{CH}_{2}\right), 62.2\left(\mathrm{CH}_{3}\right), \quad 58.9\left(\mathrm{CH}_{2}\right), 34.7\left(\mathrm{CH}_{2}\right)$. ESI ${ }^{+}-\mathrm{HRAM}: \mathrm{m} / z$ calculated for $\left[\mathrm{C}_{20} \mathrm{H}_{23} \mathrm{~N}_{4} \mathrm{O}_{2}\right]^{+}\left([\mathrm{M}+\mathrm{H}]^{+}\right)$351.181552, found 351.181459. IR (neat): $v_{\max } 3381,3061,3030$, 2934, 2891, 1545, 1509, 1445, 1319, 1039, 1002, 907, 734, 691. 
Following the general procedure $\mathrm{C}$ from 3-(hept-1-en-1-yl)-5-phenyl-1,2,4-triazine 1e (25.3 mg, $0.10 \mathrm{mmol}) \mathrm{N}$-methoxybenzylamine $2 \mathbf{d}(19.8 \mu \mathrm{L}, 0.15 \mathrm{mmol}, 1.5$ equiv) in 72 hours, the title compound (31 mg, 79\% yield) was obtained as a yellow oil after purification by column chromatography (eluent $=9: 1$ pentane/EtOAc, $R_{f}(8: 2$ petroleum ether/EtOAc $\left.)=0.6\right) .{ }^{1} \mathrm{H}$ NMR (400 MHz, chloroform- $d$ ): $\delta_{\mathrm{H}} 9.54(\mathrm{~s}, 1 \mathrm{H}), 8.15-8.11(\mathrm{~m}, 2 \mathrm{H}), 7.63-7.51(\mathrm{~m}, 3 \mathrm{H})$, $7.27-7.15(\mathrm{~m}, 5 \mathrm{H}), 3.96(\mathrm{~d}, J=13.2 \mathrm{~Hz}, 1 \mathrm{H}), 3.91(\mathrm{~d}, J=13.2 \mathrm{~Hz}, 1 \mathrm{H}), 3.66-3.51(\mathrm{~m}, 2 \mathrm{H})$, $3.27(\mathrm{dd}, J=12.8,5.2 \mathrm{~Hz}, 1 \mathrm{H}), 3.24(\mathrm{~s}, 3 \mathrm{H}), 1.89-1.74(\mathrm{~m}, 1 \mathrm{H}), 1.59-1.47(\mathrm{~m}, 2 \mathrm{H}), 1.47-$ $1.38(\mathrm{~m}, 1 \mathrm{H}), 1.28(\mathrm{~m}, 4 \mathrm{H}), 0.87(\mathrm{t}, J=6.8 \mathrm{~Hz}, 3 \mathrm{H}) .{ }^{13} \mathrm{C}\left\{{ }^{1} \mathrm{H}\right\}$ NMR (101 MHz, chloroformd): $\delta_{\mathrm{C}} 169.6(\mathrm{C}), 154.9(\mathrm{C}), 143.9(\mathrm{CH}), 138.5(\mathrm{C}), 133.9(\mathrm{C}), 132.4(\mathrm{CH}), 129.5(2 \mathrm{CH})$, 129.4 (2 CH), $128.1(2 \mathrm{CH}), 127.7(2 \mathrm{CH}), 127.1(\mathrm{CH}), 63.7(\mathrm{CH}), 61.3\left(\mathrm{CH}_{3}\right), 57.4\left(\mathrm{CH}_{2}\right)$, $38.3\left(\mathrm{CH}_{2}\right), 32.0\left(\mathrm{CH}_{2}\right), 30.4\left(\mathrm{CH}_{2}\right), 26.7\left(\mathrm{CH}_{2}\right), 22.7\left(\mathrm{CH}_{2}\right), 14.2\left(\mathrm{CH}_{3}\right)$. ESI ${ }^{+}-$HRAM: $m / z$ calculated for $\left[\mathrm{C}_{24} \mathrm{H}_{31} \mathrm{~N}_{4} \mathrm{O}\right]^{+}\left([\mathrm{M}+\mathrm{H}]^{+}\right)$391.249238, found 391.249002. IR (neat): $v_{\max } 2930$, 2857, 1544, 1508, 1496, 1318, 1047, 1001, 907, 758, 731, 690.

\section{$N$-benzyl- $N$-(1-cyclopropyl-2-(5-phenyl-1,2,4-triazin-3-yl)ethyl)- $O$ -}

methylhydroxylamine (3f). Following the general procedure A from 3-(2-cyclopropylvinyl)5-phenyl-1,2,4-triazine $\mathbf{1 f}(22 \mathrm{mg}, 0.10 \mathrm{mmol})$ and $N$-methoxybenzylamine $\mathbf{2 d}(19.8 \mu \mathrm{L}, 0.15$ mmol, 1.5 equiv) in 96 hours, the title compound (17 mg, $46 \%$ yield) was obtained as a yellow oil after purification by silica gel column chromatography (eluent $=95: 5$ $\left.\mathrm{CH}_{2} \mathrm{Cl}_{2} / \mathrm{EtOAc}, R_{f}=0.4\right) .{ }^{1} \mathrm{H}$ NMR $(400 \mathrm{MHz}$, chloroform- $d): \delta_{\mathrm{H}} 9.55(\mathrm{~s}, 1 \mathrm{H}), 8.23-8.03(\mathrm{~m}$, 2H), $7.63-7.52(\mathrm{~m}, 3 \mathrm{H}), 7.25-7.15(\mathrm{~m}, 5 \mathrm{H}), 4.13(\mathrm{~d}, J=13.1 \mathrm{~Hz}, 1 \mathrm{H}), 4.01(\mathrm{~d}, J=13.1 \mathrm{~Hz}$, 1H), $3.71(\mathrm{dd}, J=13.7,8.3 \mathrm{~Hz}, 1 \mathrm{H}), 3.44(\mathrm{dd}, J=13.7,6.1 \mathrm{~Hz}, 1 \mathrm{H}), 3.24$ (s, 3H), 2.90 (ddd, $J$ $=9.7,8.3,6.1 \mathrm{~Hz}, 1 \mathrm{H}), 1.28-1.15(\mathrm{~m}, 1 \mathrm{H}), 0.69(\mathrm{~m}, 1 \mathrm{H}), 0.48(\mathrm{~m}, 1 \mathrm{H}), 0.41(\mathrm{~m}, 1 \mathrm{H}),-0.05-$ $-0.14(\mathrm{~m}, 1 \mathrm{H}) .{ }^{13} \mathrm{C}\left\{{ }^{1} \mathrm{H}\right\}$ NMR (101 MHz, chloroform- $\left.d\right)$ : $\delta_{\mathrm{C}} 169.2(\mathrm{C}), 154.8(\mathrm{C}), 143.9(\mathrm{CH})$, $138.5(\mathrm{C}), 133.9(\mathrm{C}), 132.4(\mathrm{CH}), 129.44(2 \mathrm{CH}), 129.39(2 \mathrm{CH}), 128.1(2 \mathrm{CH}), 127.7(2 \mathrm{CH})$, 
$127.0(\mathrm{CH}), 69.4(\mathrm{CH}), 61.6\left(\mathrm{CH}_{3}\right), 58.5\left(\mathrm{CH}_{2}\right), 40.3\left(\mathrm{CH}_{2}\right), 11.6(\mathrm{CH}), 5.8\left(\mathrm{CH}_{2}\right), 2.8\left(\mathrm{CH}_{2}\right)$. ESI ${ }^{+}$-HRAM: $m / z$ calculated for $\left[\mathrm{C}_{22} \mathrm{H}_{25} \mathrm{~N}_{4} \mathrm{O}\right]^{+}\left([\mathrm{M}+\mathrm{H}]^{+}\right)$361.202288, found 361.202518. IR (neat): $v_{\max } 3030,2939,2895,1543,1507,1319,1027,691$.

\section{$N$-benzyl- $O$-methyl- $N$-(1-phenyl-3-(5-phenyl-1,2,4-triazin-3-yl)propan-2-yl)-}

hydroxylamine (3g). Following the general procedure C from 5-phenyl-3-(3-phenylprop-1en-1-yl)-1,2,4-triazine $1 \mathrm{~g}$ (27 $\mathrm{mg}, 0.10 \mathrm{mmol})$ and $N$-methoxybenzylamine $\mathbf{2 d}(19.8 \mu \mathrm{L}, 0.15$ mmol, 1.5 equiv) in 96 hours, the title compound (15.9 mg, 39\% yield) was obtained as a yellow oil after purification by silica gel column chromatography (eluent $=95: 5$ $\left.\mathrm{CH}_{2} \mathrm{Cl}_{2} / \mathrm{EtOAc}, R_{f}=0.3\right) .{ }^{1} \mathrm{H} \mathrm{NMR}\left(400 \mathrm{MHz}\right.$, chloroform- $d$ ): $\delta_{\mathrm{H}} 9.47(\mathrm{~s}, 1 \mathrm{H}), 8.08-8.01(\mathrm{~m}$, 2H), $7.62-7.49(\mathrm{~m}, 3 \mathrm{H}), 7.25-7.18(\mathrm{~m}, 4 \mathrm{H}), 7.15(\mathrm{~s}, 5 \mathrm{H}), 7.15-7.10(\mathrm{~m}, 1 \mathrm{H}), 4.06(\mathrm{~d}, J=$ $13.1 \mathrm{~Hz}, 1 \mathrm{H}), 4.02-3.92(\mathrm{~m}, 1 \mathrm{H}), 3.95(\mathrm{~d}, J=13.1 \mathrm{~Hz}, 1 \mathrm{H}), 3.55(\mathrm{dd}, J=14.2,8.3 \mathrm{~Hz}, 1 \mathrm{H})$, $3.34(\mathrm{dd}, \mathrm{J}=13.7,4.9 \mathrm{~Hz}, 1 \mathrm{H}), 3.30(\mathrm{~s}, 3 \mathrm{H}), 3.27(\mathrm{dd}, J=14.2,5.8 \mathrm{~Hz}, 1 \mathrm{H}), 2.85(\mathrm{dd}, J=$ 13.7, 9.1 Hz, 1H). ${ }^{13} \mathrm{C}\left\{{ }^{1} \mathrm{H}\right\}$ NMR (101 MHz, chloroform- $d$ ): $\delta_{\mathrm{C}} 169.1$ (C), 154.7 (C), 143.9 (CH), $139.8(\mathrm{C}), 138.0(\mathrm{C}), 133.8(\mathrm{C}), 132.4(\mathrm{CH}), 129.44(\mathrm{CH}), 129.42(\mathrm{CH}), 129.37(\mathrm{CH})$, $128.5(\mathrm{CH}), 128.2(\mathrm{CH}), 127.7(2 \mathrm{CH}), 127.2(2 \mathrm{CH}), 126.1(2 \mathrm{CH}), 65.1(\mathrm{CH}), 61.4\left(\mathrm{CH}_{3}\right)$, $57.7\left(\mathrm{CH}_{2}\right), 38.7\left(\mathrm{CH}_{2}\right), 35.7\left(\mathrm{CH}_{2}\right)$. ESI'-HRAM: $m / z$ calculated for $\left[\mathrm{C}_{26} \mathrm{H}_{27} \mathrm{~N}_{4} \mathrm{O}\right]^{+}\left([\mathrm{M}+\mathrm{H}]^{+}\right)$ 411.217938, found 411.218212. IR (neat): $v_{\max }$ 3028, 2939, 2179, 1544, 1508, 691 .

Following the general procedure $\mathrm{C}$ from 5-methyl-3-(prop-1-en-1-yl)-1,2,4-triazine $\mathbf{1 j}$ (13.5 $\mathrm{mg}, 0.10 \mathrm{mmol})$ and $N$-methoxybenzylamine $2 \mathrm{~d}(19.8 \mu \mathrm{L}, 0.15 \mathrm{mmol}, 1.5$ equiv) in 30 hours, the title compound (19.9 mg, 73\% yield) was obtained as a yellow oil after purification by column chromatography (eluent $=1: 1$ pentane/EtOAc, $R_{f}=0.4$ ). N.B.: the mixture turned from yellow to dark brown after 1-2 hours. ${ }^{1} \mathrm{H}$ NMR (400 MHz, chloroform- $d$ ): $\delta_{\mathrm{H}} 8.98$ (s, 1H), $7.29-7.19(\mathrm{~m}, 5 \mathrm{H}), 3.94(\mathrm{~d}, J=13.0 \mathrm{~Hz}, 1 \mathrm{H}), 3.79(\mathrm{~d}, J=13.0 \mathrm{~Hz}, 1 \mathrm{H}), 3.66(\mathrm{~h}, J=7.0$ $\mathrm{Hz}, 1 \mathrm{H}), 3.50(\mathrm{dd}, J=13.8,7.0 \mathrm{~Hz}, 1 \mathrm{H}), 3.24(\mathrm{~s}, 3 \mathrm{H}), 3.10(\mathrm{dd}, J=13.8,7.0 \mathrm{~Hz}, 1 \mathrm{H}),$, 
(s, 3H), $1.21(\mathrm{~d}, J=7.0 \mathrm{~Hz}, 3 \mathrm{H}) .{ }^{13} \mathrm{C}\left\{{ }^{1} \mathrm{H}\right\}$ NMR (101 MHz, chloroform- $\left.d\right): \delta_{\mathrm{C}} 168.6(\mathrm{C})$, $159.0(\mathrm{C}), 147.9(\mathrm{CH}), 138.3(\mathrm{C}), 129.6(2 \mathrm{CH}), 128.1(2 \mathrm{CH}), 127.1(\mathrm{CH}), 61.7\left(\mathrm{CH}_{3}\right), 59.4$ $(\mathrm{CH}), 58.0\left(\mathrm{CH}_{2}\right), 41.3\left(\mathrm{CH}_{2}\right), 21.9\left(\mathrm{CH}_{3}\right), 14.4\left(\mathrm{CH}_{3}\right) . \mathrm{ESI}^{+}-\mathrm{HRAM}: \mathrm{m} / z$ calculated for $\left[\mathrm{C}_{15} \mathrm{H}_{21} \mathrm{~N}_{4} \mathrm{O}\right]^{+}\left([\mathrm{M}+\mathrm{H}]^{+}\right)$273.170988, found 273.171000. IR (neat): $v_{\max }$ 2971, 2937, 2895, 1604, 1548, 1530, 1359, 1035.

\section{$N$-benzyl-O-methyl- $N$-(1-(5-(trifluoromethyl)-1,2,4-triazin-3-yl)propan-2-yl)-}

hydroxylamine (3k). Following the general procedure $\mathrm{C}$ from 5-trifluoromethyl-3-(prop-1en-1-yl)-1,2,4-triazine $1 \mathbf{k}(18.9 \mathrm{mg}, 0.10 \mathrm{mmol})$ and $N$-methoxybenzylamine $2 \mathbf{d}(19.8 \mu \mathrm{L}$, $0.15 \mathrm{mmol}, 1.5$ equiv) in 96 hours, the title compound ( $26 \mathrm{mg}, 79 \%$ yield) was obtained as a bright-yellow oil after purification by silica gel column chromatography (eluent $=95: 5$ pentane/Et $\left.2 \mathrm{O}, R_{f}=0.3\right) .{ }^{1} \mathrm{H}$ NMR $(400 \mathrm{MHz}$, chloroform- $d): \delta_{\mathrm{H}} 9.47(\mathrm{~s}, 1 \mathrm{H}), 7.25-7.12(\mathrm{~m}$, $5 \mathrm{H}), 3.95(\mathrm{~d}, J=13.0 \mathrm{~Hz}, 1 \mathrm{H}), 3.76(\mathrm{~d}, J=13.0 \mathrm{~Hz}, 1 \mathrm{H}), 3.76-3.60(\mathrm{~m}, 2 \mathrm{H}), 3.35-3.25(\mathrm{~m}$, $1 \mathrm{H}), 3.19(\mathrm{~s}, 3 \mathrm{H}), 1.28(\mathrm{~d}, J=6.2 \mathrm{~Hz}, 3 \mathrm{H}) .{ }^{13} \mathrm{C}\left\{{ }^{1} \mathrm{H}\right\} \mathrm{NMR}(101 \mathrm{MHz}$, chloroform- $d): \delta_{\mathrm{C}} 170.1$ (C), $147.3\left(\mathrm{q},{ }^{2} J_{\mathrm{C}-\mathrm{F}}=37.7 \mathrm{~Hz}, \mathrm{C}\right), 142.7\left(\mathrm{q},{ }^{3} J_{\mathrm{C}-\mathrm{F}}=2.6 \mathrm{~Hz}, \mathrm{CH}\right), 137.8(\mathrm{C}), 129.4(2 \mathrm{CH})$, $128.2(2 \mathrm{CH}), 127.3(\mathrm{CH}), 120.2\left(\mathrm{q},{ }^{1} J_{\mathrm{C}-\mathrm{F}}=276.0 \mathrm{~Hz}, \mathrm{CF}_{3}\right), 61.3\left(\mathrm{CH}_{3}\right), 59.3(\mathrm{CH}), 57.8$ $\left(\mathrm{CH}_{2}\right), 41.4(\mathrm{CH} 2), 14.4\left(\mathrm{CH}_{3}\right) . \mathrm{ESI}^{+}-\mathrm{HRAM}: \mathrm{m} / \mathrm{z}$ calculated for $\left[\mathrm{C}_{15} \mathrm{H}_{18} \mathrm{~F}_{3} \mathrm{~N}_{4} \mathrm{O}\right]^{+}\left([\mathrm{M}+\mathrm{H}]^{+}\right)$ 327.142722, found 327.142697. IR (neat): $v_{\max } 2976,2937,1334,1199,1143,1036,697$.

\section{$N$-benzyl- $N$-(1-(5,6-dimethyl-1,2,4-triazin-3-yl)propan-2-yl)- $O$-methylhydroxylamine}

(31). Following the general procedure $\mathrm{C}$ from 5,6-dimethyl-3-(prop-1-en-1-yl)-1,2,4-triazine 11 (14.9 mg, $0.10 \mathrm{mmol})$ and $N$-methoxybenzylamine $2 \mathbf{d}(19.8 \mu \mathrm{L}, 0.15 \mathrm{mmol}, 1.5$ equiv) in 72 hours, the title compound ( $25 \mathrm{mg}, 87 \%$ yield) was obtained as a yellow oil after purification by column chromatography (eluent $\left.=4: 1 \mathrm{CH}_{2} \mathrm{Cl}_{2} / \mathrm{EtOAc}, R_{f}=0.3\right) .{ }^{1} \mathrm{H} \mathrm{NMR}(400$ MHz, chloroform- $d)$ : $\delta_{\mathrm{H}} 7.28-7.21(\mathrm{~m}, 5 \mathrm{H}), 3.94(\mathrm{~d}, J=13.0 \mathrm{~Hz}, 1 \mathrm{H}), 3.80(\mathrm{~d}, J=13 \mathrm{~Hz}$, 1H), $3.63(\mathrm{~h}, J=7 \mathrm{~Hz}, 1 \mathrm{H}), 3.46(\mathrm{dd}, J=14,7 \mathrm{~Hz}, 1 \mathrm{H}), 3.26(\mathrm{~s}, 3 \mathrm{H}), 3.05(\mathrm{dd}, J=14,7 \mathrm{~Hz}$, 1H), $2.66(\mathrm{~s}, 3 \mathrm{H}), 2.47(\mathrm{~s}, 3 \mathrm{H}), 1.20(\mathrm{~d}, J=7 \mathrm{~Hz}, 3 \mathrm{H}) .{ }^{13} \mathrm{C}\left\{{ }^{1} \mathrm{H}\right\}$ NMR (101 MHz, chloroform- 
d): $\delta_{\mathrm{C}} 166.8(\mathrm{C}), 158.4(\mathrm{C}), 155.3(\mathrm{CH}), 138.4(\mathrm{C}), 129.6(2 \mathrm{CH}), 128.1(2 \mathrm{CH}), 127.1(\mathrm{CH})$, $61.8\left(\mathrm{CH}_{3}\right), 59.4(\mathrm{CH}), 58.1\left(\mathrm{CH}_{2}\right), 40.8\left(\mathrm{CH}_{2}\right), 21.8\left(\mathrm{CH}_{3}\right), 19.5\left(\mathrm{CH}_{3}\right), 14.5\left(\mathrm{CH}_{3}\right)$. ESI ${ }^{+}-$ HRAM: $m / z$ calculated for $\left[\mathrm{C}_{16} \mathrm{H}_{23} \mathrm{~N}_{4} \mathrm{O}\right]^{+}\left([\mathrm{M}+\mathrm{H}]^{+}\right)$287.186638, found 287.186558. IR (neat): $v_{\max } 3297,2972,2935,2899,1398,1039,735,698$.

$N$-(1-(1,2,4-triazin-3-yl)propan-2-yl)- $N$-benzyl- $O$-methylhydroxylamine (3m). Following the general procedure $\mathrm{C}$ from 3-(prop-1-en-1-yl)-1,2,4-triazine $1 \mathrm{~m}(12.1 \mathrm{mg}, 0.10 \mathrm{mmol})$ and $N$-methoxybenzylamine $\mathbf{2 d}(19.8 \mu \mathrm{L}, 0.15 \mathrm{mmol}, 1.5$ equiv) in 72 hours, the title compound (10 mg, 39\% yield) was obtained as a light-yellow oil after purification by silica gel column chromatography (eluent $=4: 1$ pentane/EtOAc, $\left.R_{f}=0.3\right) .{ }^{1} \mathrm{H}$ NMR $(400 \mathrm{MHz}$, chloroform- $d)$ : $\delta_{\mathrm{H}} 9.11(\mathrm{~d}, J=2.4 \mathrm{~Hz}, 1 \mathrm{H}), 8.53(\mathrm{~d}, J=2.4 \mathrm{~Hz}, 1 \mathrm{H}), 7.27-7.22(\mathrm{~m}, 5 \mathrm{H}), 3.95(\mathrm{~d}, J=13.1$ Hz, 1H), 3.79 (d, $J=13.1 \mathrm{~Hz}, 1 \mathrm{H}), 3.68(\mathrm{~h}, J=7 \mathrm{~Hz}, 1 \mathrm{H}), 3.56(\mathrm{dd}, J=13.8,7 \mathrm{~Hz}, 1 \mathrm{H}), 3.23$ (s, 3H), $3.19(\mathrm{dd}, J=13.8,7 \mathrm{~Hz}, 1 \mathrm{H}), 1.23(\mathrm{~d}, J=7 \mathrm{~Hz}, 3 \mathrm{H}) \cdot{ }^{13} \mathrm{C}\left\{{ }^{1} \mathrm{H}\right\} \mathrm{NMR}(101 \mathrm{MHz}$, chloroform- $d$ ): $\delta_{\mathrm{C}} 169.9(\mathrm{C}), 148.5(\mathrm{C}), 147.6(\mathrm{CH}), 138.2(\mathrm{C}), 129.6(2 \mathrm{CH}), 128.2(2 \mathrm{CH})$, 127.2 $(\mathrm{CH}), 61.7\left(\mathrm{CH}_{3}\right), 59.6(\mathrm{CH}), 58.1\left(\mathrm{CH}_{2}\right), 41.4\left(\mathrm{CH}_{2}\right), 14.7\left(\mathrm{CH}_{3}\right)$. ESI ${ }^{+}-\mathrm{HRAM}$ : $m / z$ calculated for $\left[\mathrm{C}_{14} \mathrm{H}_{19} \mathrm{~N}_{4} \mathrm{O}\right]^{+}\left([\mathrm{M}+\mathrm{H}]^{+}\right)$259.155338, found 259.155307. IR (neat): $v_{\max } 3406$, 2971, 2935, 2899, 1410, 1045, 735, 698.

\section{$O$-methyl- $N$-(1-(5-phenyl-1,2,4-triazin-3-yl)propan-2-yl)- $N$-(prop-2-yn-1-yl)-}

hydroxylamine (3n). Following the general procedure C from 5-phenyl-3-(prop-1-en-1-yl)1,2,4-triazine $\mathbf{1 b}(99 \mathrm{mg}, 0.5 \mathrm{mmol})$ and $N$-methoxypropargylamine $2 \mathrm{~g}(67 \mu \mathrm{L}, 0.75 \mathrm{mmol}$, 1.5 equiv) in 24 hours, the title compound ( $127 \mathrm{mg}, 90 \%$ yield) was obtained as a yellow oil after purification by silica gel column chromatography (eluent $=4: 1$ pentane/EtOAc, $R_{f}=$ 0.25). From 5-phenyl-3-(prop-1-en-1-yl)-1,2,4-triazine 1b (215 mg, $1.09 \mathrm{mmol})$ and $\mathrm{N}$ methoxypropargylamine $2 \mathrm{~g}(150 \mu \mathrm{L}, 1.69 \mathrm{mmol}, 1.5$ equiv) in 24 hours, the title compound (270 mg, 88\% yield) was obtained as a yellow oil after purification by silica gel column chromatography. ${ }^{1} \mathrm{H}$ NMR (400 MHz, chloroform- $d$ ): $\delta_{\mathrm{H}} 9.54(\mathrm{~s}, 1 \mathrm{H}), 8.21-8.17(\mathrm{~m}, 2 \mathrm{H})$, 
$7.62-7.51(\mathrm{~m}, 3 \mathrm{H}), 3.88(\mathrm{~h}, J=7 \mathrm{~Hz}, 1 \mathrm{H}), 3.77(\mathrm{dd}, J=17.0,2.5 \mathrm{~Hz}, 1 \mathrm{H}), 3.62(\mathrm{dd}, J=$ 17.0, $2.5 \mathrm{~Hz}, 1 \mathrm{H}), 3.57(\mathrm{~s}, 3 \mathrm{H}), 3.57(\mathrm{dd}, J=14.0,7.0 \mathrm{~Hz}, 1 \mathrm{H}), 3.21(\mathrm{dd}, J=14.0,7.0 \mathrm{~Hz}$, 1H), $2.20(\mathrm{t}, J=2.5 \mathrm{~Hz}, 1 \mathrm{H}), 1.19(\mathrm{~d}, J=7.0 \mathrm{~Hz}, 3 \mathrm{H}) .{ }^{13} \mathrm{C}\left\{{ }^{1} \mathrm{H}\right\}$ NMR (101 MHz, chloroformd): $\delta_{\mathrm{C}} 168.5(\mathrm{C}), 155.0(\mathrm{C}), 144.2(\mathrm{CH}), 133.8(\mathrm{C}), 132.5(\mathrm{CH}), 129.5(2 \mathrm{CH}), 127.7(2 \mathrm{CH})$, $79.1(\mathrm{C}), 72.4(\mathrm{CH}), 61.6\left(\mathrm{CH}_{3}\right), 59.1(\mathrm{CH}), 43.2\left(\mathrm{CH}_{2}\right), 41.6\left(\mathrm{CH}_{2}\right), 15.7\left(\mathrm{CH}_{3}\right)$. ESI ${ }^{+}-$ HRAM: $m / z$ calculated for $\left[\mathrm{C}_{16} \mathrm{H}_{19} \mathrm{~N}_{4} \mathrm{O}\right]^{+}\left([\mathrm{M}+\mathrm{H}]^{+}\right)$283.155338, found 283.155508. IR (neat): $v_{\max } 3291,2935,1508,1041,690$.

\section{$O$-benzyl- $N$-(1-(5-phenyl-1,2,4-triazin-3-yl)propan-2-yl)- $N$-(prop-2-yn-1-yl)-}

hydroxylamine (3o). Following the general procedure C from 5-phenyl-3-(prop-1-en-1-yl)1,2,4-triazine $\mathbf{1 b}(19.7 \mathrm{mg}, 0.10 \mathrm{mmol})$ and $O$-benzyl- $N$-propargylhydroxylamine $\mathbf{2 h}(24 \mathrm{mg}$, $0.15 \mathrm{mmol}, 1.5$ equiv) in 24 hours, the title compound ( $29.2 \mathrm{mg}, 81 \%$ yield) was obtained as a yellow oil after purification by silica gel column chromatography (eluent $=4: 1$ pentane/EtOAc, $\left.R_{f}=0.3\right) .{ }^{1} \mathrm{H}$ NMR $(400 \mathrm{MHz}$, chloroform- $d): \delta_{\mathrm{H}} 9.51(\mathrm{~s}, 1 \mathrm{H}), 8.17(\mathrm{dd}, J=$ 8.1, $1.7 \mathrm{~Hz}, 2 \mathrm{H}), 7.64-7.49$ (m, 3H), $7.38-7.24(\mathrm{~m}, 5 \mathrm{H}), 4.83$ (d, J = 10.7 Hz, 1H), 4.78 (d, $J=10.7 \mathrm{~Hz}, 1 \mathrm{H}), 3.94(\mathrm{~h}, J=7 \mathrm{~Hz}, 1 \mathrm{H}), 3.76(\mathrm{dd}, J=16.7,2.5 \mathrm{~Hz}, 1 \mathrm{H}), 3.65(\mathrm{dd}, J=16.7$, $2.5 \mathrm{~Hz}, 1 \mathrm{H}), 3.60(\mathrm{dd}, J=14.0,7 \mathrm{~Hz}, 1 \mathrm{H}), 3.25(\mathrm{dd}, J=14.0,7 \mathrm{~Hz}, 1 \mathrm{H}), 2.20(\mathrm{t}, J=2.5 \mathrm{~Hz}$, 1H), $1.23(\mathrm{~d}, J=7 \mathrm{~Hz}, 3 \mathrm{H}) .{ }^{13} \mathrm{C}\left\{{ }^{1} \mathrm{H}\right\}$ NMR (101 MHz, chloroform- $\left.d\right): \delta_{\mathrm{C}} 168.6(\mathrm{C}), 155.0$ (C), $144.1(\mathrm{CH}), 137.3(\mathrm{C}), 133.8(\mathrm{C}), 132.5(\mathrm{CH}), 129.4(2 \mathrm{CH}), 128.9(2 \mathrm{CH}), 128.4(2 \mathrm{CH})$, $128.0(\mathrm{CH}), 127.7(2 \mathrm{CH}), 79.5(\mathrm{C}), 76.3\left(\mathrm{CH}_{2}\right), 72.4(\mathrm{CH}), 59.5(\mathrm{CH}), 43.8\left(\mathrm{CH}_{2}\right), 41.4$ $\left(\mathrm{CH}_{2}\right), 15.7\left(\mathrm{CH}_{3}\right)$. ESI ${ }^{+}-\mathrm{HRAM}: m / z$ calculated for $\left[\mathrm{C}_{22} \mathrm{H}_{23} \mathrm{~N}_{4} \mathrm{O}\right]^{+}\left([\mathrm{M}+\mathrm{H}]^{+}\right) 359.186638$, found 359.186831. IR (neat): $v_{\max } 3293,2973,2875,1601,1544,1508,691$.

$O$-benzyl- $N$-(1-(5-phenyl-1,2,4-triazin-3-yl)propan-2-yl)hydroxylamine (3p). Following the general procedure A from 5-phenyl-3-(prop-1-en-1-yl)-1,2,4-triazine 1b (19.7 mg, 0.10 mmol) and $O$-benzylhydroxylamine $2 \mathbf{i}(16 \mu \mathrm{L}, 0.15 \mathrm{mmol}, 1.5$ equiv) in 24 hours, the title compound (29.1 mg, 91\% yield) was obtained as a yellow oil after purification by silica gel 
column chromatography (eluent $=65: 35$ petroleum ether/EtOAc, $\left.R_{f}=0.3\right) .{ }^{1} \mathrm{H}$ NMR $(400$ $\mathrm{MHz}$, chloroform- $d): \delta_{\mathrm{H}} 9.52(\mathrm{~s}, 1 \mathrm{H}), 8.22-8.09(\mathrm{~m}, 2 \mathrm{H}), 7.65-7.50(\mathrm{~m}, 3 \mathrm{H}), 7.39-7.20$ (m, 5H), $4.71(\mathrm{~s}, 2 \mathrm{H}), 3.87-3.74(\mathrm{~m}, 1 \mathrm{H}), 3.43(\mathrm{dd}, J=14.5,7.3 \mathrm{~Hz}, 1 \mathrm{H}), 3.24(\mathrm{dd}, J=14.5$, $5.5 \mathrm{~Hz}, 1 \mathrm{H}), 1.24(\mathrm{~d}, J=6.5 \mathrm{~Hz}, 3 \mathrm{H}) .{ }^{13} \mathrm{C}\left\{{ }^{1} \mathrm{H}\right\} \mathrm{NMR}(101 \mathrm{MHz}$, chloroform- $d): \delta_{\mathrm{C}} 168.3(\mathrm{C})$, $155.1(\mathrm{C}), 144.3(\mathrm{CH}), 138.0(\mathrm{C}), 133.7(\mathrm{C}), 132.6(\mathrm{CH}), 129.5(2 \mathrm{CH}), 128.40(2 \mathrm{CH})$, $128.39(2 \mathrm{CH}), 127.8(\mathrm{CH}), 127.7(2 \mathrm{CH}), 76.7\left(\mathrm{CH}_{2}\right), 55.5(\mathrm{CH}), 41.8\left(\mathrm{CH}_{2}\right), 18.5\left(\mathrm{CH}_{3}\right)$. ESI ${ }^{+}-H R A M: ~ m / z$ calculated for $\left[\mathrm{C}_{19} \mathrm{H}_{21} \mathrm{~N}_{4} \mathrm{O}\right]^{+}\left([\mathrm{M}+\mathrm{H}]^{+}\right)$321.170988, found 321.171098. IR (neat): $v_{\max } 2968,2910,1601,1544,1508$.

\section{$O$-methyl- $N$-(2-(5-phenyl-1,2,4-triazin-3-yl)ethyl)- $N$-(prop-2-yn-1-yl)hydroxylamine (3q).} $\mathrm{N}$-methoxypropargylamine hydrochloride $2 \mathrm{~g} . \mathrm{HCl}$ (845 mg, $6.96 \mathrm{mmol}, 1.5$ equiv) was added into a mixture of 5-phenyl-3-vinyl-1,2,4-triazine 1a $(850 \mathrm{mg}, 4.64 \mathrm{mmol})$ and sodium bicarbonate (585 mg, $6.96 \mathrm{mmol}, 1.5$ equiv) in $50 \mathrm{~mL}$ of tetrahydrofuran. The mixture was stirred at room temperature for 24 hours (it turned brown after a few hours). $50 \mathrm{~mL}$ of a $0.3 \mathrm{M}$ sodium bicarbonate aqueous solution were then added, and the mixture was extracted with dichloromethane $(3 \times 50 \mathrm{~mL})$. The combined organic extracts were dried over magnesium sulfate and concentrated on rotary evaporator. Purification by silica gel column chromatography (eluent $=8: 2$ petroleum ether/EtOAc, $R_{f}=0.3$ ) afforded the title compound

(960 mg, $3.58 \mathrm{mmol}, 77 \%$ yield) as a yellow solid. ${ }^{1} \mathrm{H}$ NMR (400 MHz, chloroform- $d$ ): $\delta_{\mathrm{H}}$ $9.55(\mathrm{~s}, 1 \mathrm{H}), 8.21-8.16(\mathrm{~m}, 2 \mathrm{H}), 7.63-7.52(\mathrm{~m}, 3 \mathrm{H}), 3.64(\mathrm{~d}, J=2.5 \mathrm{~Hz}, 2 \mathrm{H}), 3.56(\mathrm{~s}, 3 \mathrm{H})$, $3.48-3.44(\mathrm{~m}, 4 \mathrm{H}), 2.25(\mathrm{t}, J=2.5 \mathrm{~Hz}, 1 \mathrm{H}) .{ }^{13} \mathrm{C}\left\{{ }^{1} \mathrm{H}\right\}$ NMR $(101 \mathrm{MHz}$, chloroform- $d): \delta_{\mathrm{C}}$ $168.4(\mathrm{C}), 155.0(\mathrm{C}), 144.2(\mathrm{CH}), 133.6(\mathrm{C}), 132.4(\mathrm{CH}), 129.4(2 \mathrm{CH}), 127.6(2 \mathrm{CH}), 78.4$ (C), 73.0(CH), $61.1\left(\mathrm{CH}_{3}\right), 55.4\left(\mathrm{CH}_{2}\right), 46.5\left(\mathrm{CH}_{2}\right), 35.3\left(\mathrm{CH}_{2}\right)$. ESI ${ }^{+}-H R A M: m / z$ calcd for $\left[\mathrm{C}_{15} \mathrm{H}_{17} \mathrm{~N}_{4} \mathrm{O}\right]^{+}\left([\mathrm{M}+\mathrm{H}]^{+}\right) 269.139688$, found $269.139649 ; \mathrm{m} / \mathrm{z}$ calcd for $\left[\mathrm{C}_{15} \mathrm{H}_{16} \mathrm{~N}_{4} \mathrm{NaO}\right]^{+}$ $\left([\mathrm{M}+\mathrm{Na}]^{+}\right)$291.121632, found 291.121608. IR (neat): $v_{\max } 3263,2931,2859,1552,1517$, $1319,1044,1032,1000,686,667 . \mathrm{mp}=57-59^{\circ} \mathrm{C}$. 
6-methoxy-2-phenyl-5,6,7,8-tetrahydro-1,6-naphthyridine (4a). A solution of triazine 3q (440 mg, $1.64 \mathrm{mmol}$ ) in $16 \mathrm{~mL}$ of $\alpha, \alpha, \alpha$-trifluorotoluene was heated at $180{ }^{\circ} \mathrm{C}$ under microwaves irradiation for 2 hours. The mixture was then concentrated on rotary evaporator and the product was purified by silica gel column chromatography (eluent $=4: 1$ petroleum ether/EtOAc, $\left.R_{f}=0.4\right)$ to afford the expected tetrahydro-[1,6]-naphthyridine $(320 \mathrm{mg}, 81 \%$ yield) as a beige solid. ${ }^{1} \mathrm{H}$ NMR $\left(250 \mathrm{MHz}\right.$, dimethylsulfoxide- $\left.d_{6}, 80{ }^{\circ} \mathrm{C}\right): \delta_{\mathrm{H}} 8.08-7.96(\mathrm{~m}$, 2H), $7.69(\mathrm{~d}, J=8.0 \mathrm{~Hz}, 1 \mathrm{H}), 7.56(\mathrm{~d}, J=8.0 \mathrm{~Hz}, 1 \mathrm{H}), 7.54-7.35(\mathrm{~m}, 3 \mathrm{H}), 4.10(\mathrm{~s}, 2 \mathrm{H}), 3.55$ $(\mathrm{d}, J=0.4 \mathrm{~Hz}, 3 \mathrm{H}), 3.30(\mathrm{t}, J=6.2 \mathrm{~Hz}, 2 \mathrm{H}), 3.04(\mathrm{t}, J=6.2 \mathrm{~Hz}, 2 \mathrm{H}) .{ }^{13} \mathrm{C}\left\{{ }^{1} \mathrm{H}\right\} \mathrm{NMR}(101$ $\mathrm{MHz}$, dimethylsulfoxide- $\left.d_{6}\right): \delta_{\mathrm{C}} 153.9(\mathrm{C}), 153.5(\mathrm{C}), 138.6(\mathrm{C}), 135.9(\mathrm{CH}), 128.7(\mathrm{CH})$, $128.6(2 \mathrm{CH}), 127.4(\mathrm{C}), 126.4(2 \mathrm{CH}), 117.7(\mathrm{CH}), 58.7\left(\mathrm{CH}_{3}\right), 55.4\left(\mathrm{CH}_{2}\right), 51.4\left(\mathrm{CH}_{2}\right), 30.1$ $\left(\mathrm{CH}_{2}\right)$. ESI ${ }^{+}-\mathrm{HRAM}: \mathrm{m} / \mathrm{z}$ calculated for $\left[\mathrm{C}_{15} \mathrm{H}_{17} \mathrm{~N}_{2} \mathrm{O}\right]^{+}\left([\mathrm{M}+\mathrm{H}]^{+}\right)$241.133540, found 241.133561. IR (neat): $v_{\max } 2969,2933,2888,2831,2809,1568,1458,1038,774,735,694$. $\mathrm{mp}=62-64{ }^{\circ} \mathrm{C}$.

General procedure $D$ for the one-pot synthesis of $N$-methoxytetrahydro-[1,6]naphthyridines (4). $N$-methoxy propargylamines $2 \mathbf{g}$-h (1.5 equiv) were added to a solution of 3-vinyl-1,2,4-triazines 1a-l (0.3 mmol, $0.1 \mathrm{M})$ and trifluoromethanesulfonimide E (20 mol\%) in $\alpha, \alpha, \alpha$-trifluorotoluene in a microwaves vial. The mixture was stirred at room temperature until TLC monitoring revealed a complete transformation of starting material 1. Potassium carbonate ( 1 equiv) was then added, the vial was sealed and heated at $180{ }^{\circ} \mathrm{C}$ under microwaves irradiation (gradient temperature from room temperature to $180{ }^{\circ} \mathrm{C}$ over 5 minutes) for 2 hours. At room temperature, the reaction mixture was subsequently filtered through cotton and purified by silica gel column chromatography to afford the expected tetrahydronaphthyridine 4. Remark: NMR spectra for tetrahydro-[1,6]-naphthyridines were carried out at $80{ }^{\circ} \mathrm{C}$ in dimethylsulfoxide- $d_{6}$ in order to overcome the broad signal issues observed for the aliphatic cycle. We considered the difference of chemical shifts values for 
tetramethylsilane between 25 and $80{ }^{\circ} \mathrm{C}$ insignificant. ${ }^{32}$ Chemical shifts were consequently calibrated on dimethylsulfoxide signals ( $\delta=2.50$ ppm for ${ }^{1} \mathrm{H}$ and $\delta=39.52 \mathrm{ppm}$ for ${ }^{13} \mathrm{C}$ ).

6-Methoxy-7-methyl-2-phenyl-5,6,7,8-tetrahydro-1,6-naphthyridine (4b). Following the general procedure D from 5-phenyl-3-(prop-1-en-1-yl)-1,2,4-triazine $\mathbf{1 b}$ (59 mg, $0.30 \mathrm{mmol}$ ) and $N$-methoxypropargylamine $2 \mathrm{~g}(40 \mu \mathrm{L}, 0.45 \mathrm{mmol}, 1.5$ equiv.) for 24 hours at room temperature (TLC monitoring in 8:2 petroleum ether/AcOEt), the title compound was obtained as a yellow solid (71 mg, 93\%) after column chromatography (8:2 pentane/AcOEt, $\left.R_{f}=0.3\right) .{ }^{1} \mathrm{H}$ NMR $\left(250 \mathrm{MHz}\right.$, dimethylsulfoxide- $\left.d_{6}, 80{ }^{\circ} \mathrm{C}\right): \delta_{\mathrm{H}} 8.05-7.96(\mathrm{~m}, 2 \mathrm{H}), 7.68(\mathrm{~d}, J$ $=8.0 \mathrm{~Hz}, 1 \mathrm{H}), 7.56(\mathrm{~d}, J=8.0 \mathrm{~Hz}, 1 \mathrm{H}), 7.51-7.39(\mathrm{~m}, 3 \mathrm{H}), 4.32(\mathrm{~d}, J=16.0 \mathrm{~Hz}, 1 \mathrm{H}), 4.00(\mathrm{~d}$, $J=16.0 \mathrm{~Hz}, 1 \mathrm{H}), 3.55(\mathrm{~s}, 3 \mathrm{H}), 3.25(\mathrm{dqd}, J=9.6,6.3,4.9 \mathrm{~Hz}, 1 \mathrm{H}), 2.97(\mathrm{dd}, J=17.2,4.9 \mathrm{~Hz}$ 1H), $2.82(\mathrm{dd}, J=17.2,9.6 \mathrm{~Hz}, 1 \mathrm{H}), 1.28(\mathrm{~d}, J=6.3 \mathrm{~Hz}, 3 \mathrm{H}) .{ }^{13} \mathrm{C}\left\{{ }^{1} \mathrm{H}\right\} \mathrm{NMR}(63 \mathrm{MHz}$, dimethylsulfoxide- $\left.d_{6}, 80{ }^{\circ} \mathrm{C}\right): \delta_{\mathrm{C}} 153.7(\mathrm{C}), 153.4(\mathrm{C}), 138.5(\mathrm{C}), 135.0(\mathrm{CH}), 128.1(\mathrm{CH})$, $128.1(2 \mathrm{CH}), 126.9(\mathrm{C}), 126.0(2 \mathrm{CH}), 117.2(\mathrm{CH}), 59.8\left(\mathrm{CH}_{3}\right), 56.2(\mathrm{CH}), 53.8\left(\mathrm{CH}_{2}\right), 36.4$ $\left(\mathrm{CH}_{2}\right), 18.0\left(\mathrm{CH}_{3}\right)$. ESI ${ }^{+}-\mathrm{HRAM}: m / z$ calculated for $\left[\mathrm{C}_{16} \mathrm{H}_{19} \mathrm{~N}_{2} \mathrm{O}\right]^{+}\left([\mathrm{M}+\mathrm{H}]^{+}\right)$255.149190, found 255.149401. IR (neat): $v_{\max } 2981,2941,2843,1463,1449,1028,776,695 . \mathrm{mp}=65-66$ ${ }^{\circ} \mathrm{C}$.

6-methoxy-8-methyl-2-phenyl-5,6,7,8-tetrahydro-1,6-naphthyridine (4c). Following the general procedure D from 5-phenyl-3-(prop-1-en-2-yl)-1,2,4-triazine $1 \mathbf{c}$ (59 mg, $0.30 \mathrm{mmol})$ and $N$-methoxypropargylamine $2 \mathrm{~g}(40 \mu \mathrm{L}, 0.45 \mathrm{mmol}, 1.5$ equiv) for 24 hours at room temperature (TLC monitoring in 4:1 petroleum ether/EtOAc), the title compound was obtained as a brown oil (49 mg, 64\% yield) after silica gel column chromatography (eluent $=$ 4:1 petroleum ether/Et $\left.{ }_{2} \mathrm{O}, R_{f}=0.2\right) .{ }^{1} \mathrm{H}$ NMR $\left(250 \mathrm{MHz}\right.$, dimethylsulfoxide- $\left.d_{6}, 80{ }^{\circ} \mathrm{C}\right): \delta_{\mathrm{H}}$ $8.12-7.99(\mathrm{~m}, 2 \mathrm{H}), 7.69(\mathrm{~d}, \mathrm{~J}=8.0 \mathrm{~Hz}, 1 \mathrm{H}), 7.55(\mathrm{~d}, \mathrm{~J}=8.0 \mathrm{~Hz} 1 \mathrm{H}), 7.55-7.35(\mathrm{~m}, 3 \mathrm{H})$, $4.13(\mathrm{~d}, \mathrm{~J}=14.8 \mathrm{~Hz}, 1 \mathrm{H}), 4.04(\mathrm{~d}, \mathrm{~J}=14.8 \mathrm{~Hz}, 1 \mathrm{H}), 3.55(\mathrm{~s}, 3 \mathrm{H}), 3.43(\mathrm{dd}, \mathrm{J}=11.0,5.5 \mathrm{~Hz}$, $1 \mathrm{H}), 3.31-3.10(\mathrm{~m}, 1 \mathrm{H}), 2.90(\mathrm{dd}, \mathrm{J}=11.0,7.7 \mathrm{~Hz}, 1 \mathrm{H}), 1.43(\mathrm{~d}, \mathrm{~J}=7.0 \mathrm{~Hz}, 3 \mathrm{H}) .{ }^{13} \mathrm{C}\left\{{ }^{1} \mathrm{H}\right\}$ 
NMR (63 MHz, dimethylsulfoxide- $\left.d_{6}, 80^{\circ} \mathrm{C}\right): \delta_{\mathrm{C}} 157.0(\mathrm{C}), 153.8(\mathrm{C}), 138.5(\mathrm{C}), 135.3(\mathrm{CH})$, $128.2(\mathrm{CH}), 128.1(2 \mathrm{CH}), 126.4(\mathrm{CH}), 125.9(2 \mathrm{CH}), 117.2(\mathrm{CH}), 59.0\left(\mathrm{CH}_{2}\right), 58.2\left(\mathrm{CH}_{3}\right)$, $56.03\left(\mathrm{CH}_{2}\right), 34.5(\mathrm{CH}), 18.1\left(\mathrm{CH}_{3}\right)$. ESI ${ }^{+}-\mathrm{HRAM}: \mathrm{m} / z$ calculated for $\left[\mathrm{C}_{16} \mathrm{H}_{19} \mathrm{~N}_{2} \mathrm{O}\right]^{+}\left([\mathrm{M}+\mathrm{H}]^{+}\right)$ 255.149190, found 255.149032. IR (neat): $v_{\max } 2931,2809,1458,1048,762,692$.

(6-methoxy-2-phenyl-5,6,7,8-tetrahydro-1,6-naphthyridin-7-yl)methanol (4d). Following the general procedure D from 3-(5-phenyl-1,2,4-triazin-3-yl)prop-2-en-1-ol 1d (64 mg, 0.30 mmol) and $N$-methoxypropargylamine $2 \mathrm{~g}$ ( $40 \mu \mathrm{L}, 0.45 \mathrm{mmol}, 1.5$ equiv) for 24 hours at room temperature (TLC monitoring in 1:1 petroleum ether/ EtOAc, N.B.: the mixture initially heterogeneous became limpid after 4 hours), the title compound was obtained as a brown solid (62 mg, 76\% yield) after silica gel column chromatography (eluent $=1: 4$ petroleum ether/EtOAc, $\left.R_{f}=0.2\right) .{ }^{1} \mathrm{H}$ NMR $\left(250 \mathrm{MHz}\right.$, dimethylsulfoxide- $\left.d_{6}, 80{ }^{\circ} \mathrm{C}\right): \delta_{\mathrm{H}} 8.09-7.96(\mathrm{~m}$, 2H), $7.68(\mathrm{~d}, J=8.0 \mathrm{~Hz}, 1 \mathrm{H}), 7.55(\mathrm{dt}, J=8.0,0.8 \mathrm{~Hz}, 1 \mathrm{H}), 7.51-7.35(\mathrm{~m}, 3 \mathrm{H}), 4.35$ (br s, $1 \mathrm{H}), 4.33(\mathrm{~d}, J=16.4 \mathrm{~Hz}, 1 \mathrm{H}), 4.04(\mathrm{dd}, J=16.4,0.8 \mathrm{~Hz}, 1 \mathrm{H}), 3.80(\mathrm{dd}, J=10.7,5.3 \mathrm{~Hz}$, $1 \mathrm{H}), 3.65-3.53(\mathrm{~m}, 1 \mathrm{H}), 3.53(\mathrm{~s}, 3 \mathrm{H}), 3.27-3.14(\mathrm{~m}, 1 \mathrm{H}), 3.09-2.82(\mathrm{~m}, 2 \mathrm{H}) .{ }^{13} \mathrm{C}\left\{{ }^{1} \mathrm{H}\right\}$ NMR (63 MHz, dimethylsulfoxide- $\left.d_{6}, 80^{\circ} \mathrm{C}\right): \delta_{\mathrm{C}} 153.7(\mathrm{C}), 153.6(\mathrm{C}), 138.6(\mathrm{C}), 135.0(\mathrm{CH})$, $128.1(\mathrm{CH}), 128.1(2 \mathrm{CH}), 127.0(\mathrm{C}), 126.0(2 \mathrm{CH}), 117.2(\mathrm{CH}), 62.5(\mathrm{CH}), 62.1\left(\mathrm{CH}_{2}\right), 59.5$ $\left(\mathrm{CH}_{3}\right), 54.0\left(\mathrm{CH}_{2}\right), 31.1\left(\mathrm{CH}_{2}\right)$. ESI'-HRAM: $m / z$ calculated for $\left[\mathrm{C}_{16} \mathrm{H}_{19} \mathrm{~N}_{2} \mathrm{O}_{2}\right]^{+}\left([\mathrm{M}+\mathrm{H}]^{+}\right)$ 271.144104, found 271.144071. IR (neat): $v_{\max } 3250,2937,2360,1459,1071,1037,770$, 687. $\mathrm{mp}=109-111^{\circ} \mathrm{C}$.

6-methoxy-7-pentyl-2-phenyl-5,6,7,8-tetrahydro-1,6-naphthyridine (4e). Following the general procedure D from 3-(hept-1-en-1-yl)-5-phenyl-1,2,4-triazine 1e (76 mg, $0.30 \mathrm{mmol})$ and $N$-methoxypropargylamine $2 \mathrm{~g}(40 \mu \mathrm{L}, 0.45 \mathrm{mmol}, 1.5$ equiv) for 96 hours at room temperature (TLC monitoring in 9:1 petroleum ether/EtOAc), the title compound was obtained as a light-brown solid (77 mg, 83\% yield) after silica gel column chromatography (eluent $=$ 95:5 petroleum ether $/$ EtOAc, $R_{f}=0.3$ ). From 3-(hept-1-en-1-yl)-5-phenyl-1,2,4- 
triazine $1 \mathrm{e}(253 \mathrm{mg}, 1.0 \mathrm{mmol})$ and $N$-methoxypropargylamine $2 \mathrm{~g}(133 \mu \mathrm{L}, 1.5 \mathrm{mmol}, 1.5$ equiv) for 96 hours at room temperature, the title compound was obtained as a light-brown solid (223 mg, $0.72 \mathrm{mmol}, 72 \%$ yield) after silica gel column chromatography. ${ }^{1} \mathrm{H}$ NMR (250 $\mathrm{MHz}$, dimethylsulfoxide- $\left.d_{6}, 80{ }^{\circ} \mathrm{C}\right): \delta_{\mathrm{H}} 8.08-7.96(\mathrm{~m}, 2 \mathrm{H}), 7.68(\mathrm{~d}, J=7.99,1 \mathrm{H}), 7.55(\mathrm{dt}, J$ $=8.0,1.0 \mathrm{~Hz}, 1 \mathrm{H}), 7.54-7.32(\mathrm{~m}, 3 \mathrm{H}), 4.32(\mathrm{~d}, J=16.8 \mathrm{~Hz}, 1 \mathrm{H}), 4.05(\mathrm{dd}, J=16.8,1.0 \mathrm{~Hz}$, $1 \mathrm{H}), 3.52(\mathrm{~s}, 3 \mathrm{H}), 3.16-3.04(\mathrm{~m}, 2 \mathrm{H}), 3.00-2.69(\mathrm{~m}, 2 \mathrm{H}), 1.86-1.70(\mathrm{~m}, 1 \mathrm{H}), 1.50(\mathrm{~m}$, 2H), $1.45-1.21(\mathrm{~m}, 4 \mathrm{H}), 0.98-0.83(\mathrm{~m}, 3 \mathrm{H}) .{ }^{13} \mathrm{C}\left\{{ }^{1} \mathrm{H}\right\}$ NMR (63 MHz, dimethylsulfoxide$\left.d_{6}, 80{ }^{\circ} \mathrm{C}\right): \delta_{\mathrm{C}} 153.7(\mathrm{C}), 153.6(\mathrm{C}), 138.6(\mathrm{C}), 134.9(\mathrm{CH}), 128.1(\mathrm{CH}), 128.0(2 \mathrm{CH}), 127.0$ (C), $125.9(2 \mathrm{CH}), 117.2(\mathrm{CH}), 60.3(\mathrm{CH}), 59.4\left(\mathrm{CH}_{3}\right), 53.6\left(\mathrm{CH}_{2}\right), 33.3\left(\mathrm{CH}_{2}\right), 32.1\left(\mathrm{CH}_{2}\right)$, $31.0\left(\mathrm{CH}_{2}\right), 24.8\left(\mathrm{CH}_{2}\right), 21.6\left(\mathrm{CH}_{2}\right), 13.3\left(\mathrm{CH}_{3}\right) . \quad \mathrm{ESI}^{+}-\mathrm{HRAM}: \mathrm{m} / \mathrm{z}$ calculated for $\left[\mathrm{C}_{20} \mathrm{H}_{27} \mathrm{~N}_{2} \mathrm{O}\right]^{+}\left([\mathrm{M}+\mathrm{H}]^{+}\right)$311.211790, found 311.211662. IR (neat): $v_{\max }$ 2931, 2809, 2359, $1586,1458,1048,762,692 . \mathrm{mp}=45-46^{\circ} \mathrm{C}$.

7-cyclopropyl-6-methoxy-2-phenyl-5,6,7,8-tetrahydro-1,6-naphthyridine (4f). Following the general procedure D from 3-(2-cyclopropylvinyl)-5-phenyl-1,2,4-triazine 1e (67 mg, 0.30 mmol) and $N$-methoxypropargylamine $2 \mathrm{~g}$ ( $40 \mu \mathrm{L}, 0.45 \mathrm{mmol}, 1.5$ equiv) for 96 hours at room temperature (TLC monitoring in 4:1 petroleum ether/EtOAc), the title compound was obtained as a light-brown solid (67 mg, 80\% yield) after silica gel column chromatography (eluent $=9: 1$ pentane/EtOAc, $\left.R_{f}=0.3\right) .{ }^{1} \mathrm{H}$ NMR $\left(250 \mathrm{MHz}\right.$, dimethylsulfoxide- $\left.d_{6}, 80{ }^{\circ} \mathrm{C}\right): \delta_{\mathrm{H}}$ $8.02-7.94(\mathrm{~m}, 2 \mathrm{H}), 7.63(\mathrm{~d}, J=7.9 \mathrm{~Hz}, 1 \mathrm{H}), 7.50(\mathrm{dt}, J=8.1,0.8 \mathrm{~Hz}, 1 \mathrm{H}), 7.47-7.30(\mathrm{~m}$, $3 \mathrm{H}), 4.30(\mathrm{~d}, J=16.5 \mathrm{~Hz}, 1 \mathrm{H}), 3.97(\mathrm{dd}, J=16.5,0.8 \mathrm{~Hz}, 1 \mathrm{H}), 3.55(\mathrm{~s}, 3 \mathrm{H}), 2.93(\mathrm{~d}, J=7.2$ $\mathrm{Hz}, 2 \mathrm{H}), 2.54-2.37(\mathrm{~m}, 1 \mathrm{H}), 1.06-0.91(\mathrm{~m}, 1 \mathrm{H}), 0.60-0.45(\mathrm{~m}, 2 \mathrm{H}), 0.45-0.34(\mathrm{~m}, 1 \mathrm{H})$, $0.28-0.16(\mathrm{~m}, 1 \mathrm{H}) .{ }^{13} \mathrm{C}\left\{{ }^{1} \mathrm{H}\right\}$ NMR $\left(63 \mathrm{MHz}\right.$, dimethylsulfoxide- $\left.d_{6}, 80{ }^{\circ} \mathrm{C}\right): \delta_{\mathrm{C}} 153.7(\mathrm{C})$, $153.5(\mathrm{C}), 138.5(\mathrm{C}), 134.9(\mathrm{CH}), 128.12(\mathrm{CH}), 128.07(2 \mathrm{CH}), 127.0(\mathrm{C}), 125.9(2 \mathrm{CH})$, 117.2 (CH), $64.7(\mathrm{CH}), 59.6\left(\mathrm{CH}_{3}\right), 53.9\left(\mathrm{CH}_{2}\right), 33.4\left(\mathrm{CH}_{2}\right), 13.6(\mathrm{CH}), 3.6\left(\mathrm{CH}_{2}\right), 1.7\left(\mathrm{CH}_{2}\right)$. ESI'-HRAM: $m / z$ calculated for $\left[\mathrm{C}_{18} \mathrm{H}_{21} \mathrm{~N}_{2} \mathrm{O}\right]^{+}\left([\mathrm{M}+\mathrm{H}]^{+}\right)$281.164840, found 281.164805. 
IR (neat): $\nu_{\max } 3068,2926,2906,1567,1456,1423,1036,769,689 . \mathrm{mp}=85-86^{\circ} \mathrm{C}$.

7-benzyl-6-methoxy-2-phenyl-5,6,7,8-tetrahydro-1,6-naphthyridine (4g). Following the general procedure D from 5-phenyl-3-(3-phenylprop-1-en-1-yl)-1,2,4-triazine $\mathbf{1 g}$ (82 mg, 0.30 mmol) and $N$-methoxypropargylamine $2 \mathrm{~g}$ (40 $\mu \mathrm{L}, 0.45 \mathrm{mmol}, 1.5$ equiv) for 65 hours at room temperature (TLC monitoring in 4:1 petroleum ether/EtOAc), the title compound was obtained as a brown solid (77 mg, 78\% yield) after silica gel column chromatography (eluent $=9: 1$ pentane/EtOAc, $\left.R_{f}=0.5\right) .{ }^{1} \mathrm{H}$ NMR $\left(250 \mathrm{MHz}\right.$, dimethylsulfoxide- $\left.d_{6}, 80{ }^{\circ} \mathrm{C}\right): \delta_{\mathrm{H}} 8.03-$ $7.95(\mathrm{~m}, 2 \mathrm{H}), 7.68(\mathrm{~d}, J=8.0,1 \mathrm{H}), 7.57(\mathrm{~d}, J=8.0,1 \mathrm{H}), 7.50-7.35(\mathrm{~m}, 3 \mathrm{H}), 7.35-7.29(\mathrm{~m}$, 4H), $7.28-7.14(\mathrm{~m}, 1 \mathrm{H}), 4.37(\mathrm{~d}, J=16.5 \mathrm{~Hz}, 1 \mathrm{H}), 4.09$ (d, $J=16.5 \mathrm{~Hz}, 1 \mathrm{H}), 3.58(\mathrm{~s}, 3 \mathrm{H})$, 3.47 (ddt, $J=9.9,8.3,5.5 \mathrm{~Hz}, 1 \mathrm{H}), 3.22(\mathrm{dd}, J=13.5,5.5 \mathrm{~Hz}, 1 \mathrm{H}), 2.91(\mathrm{dd}, J=17.0,9.9 \mathrm{~Hz}$, 1H), $2.76(\mathrm{dd}, J=13.5,8.3 \mathrm{~Hz}, 1 \mathrm{H}), 2.72(\mathrm{dd}, J=17.0,5.5 \mathrm{~Hz}, 1 \mathrm{H}) .{ }^{13} \mathrm{C}\left\{{ }^{1} \mathrm{H}\right\} \mathrm{NMR}(63 \mathrm{MHz}$, dimethylsulfoxide- $\left.d_{6}, 80^{\circ} \mathrm{C}\right): \delta_{\mathrm{C}} 153.7(\mathrm{C}), 153.4(\mathrm{C}), 138.8(\mathrm{CH}), 138.5(\mathrm{CH}), 135.0(\mathrm{CH})$, $128.7(2 \mathrm{CH}), 128.11(\mathrm{CH}), 128.06(2 \mathrm{CH}), 127.8(2 \mathrm{CH}), 126.8(\mathrm{C}), 126.0(2 \mathrm{CH}), 125.5$ (CH), $117.3(\mathrm{CH}), 61.8(\mathrm{CH}), 59.5\left(\mathrm{CH}_{3}\right), 53.6\left(\mathrm{CH}_{2}\right), 38.4\left(\mathrm{CH}_{2}\right), 33.1\left(\mathrm{CH}_{2}\right)$. ESI ${ }^{+}-\mathrm{HRAM}_{\text {: }}$ $m / z$ calculated for $\left[\mathrm{C}_{22} \mathrm{H}_{23} \mathrm{~N}_{2} \mathrm{O}\right]^{+}\left([\mathrm{M}+\mathrm{H}]^{+}\right)$331.180490, found 331.181164. IR (neat): $v_{\max }$ $2926,1716,1586,1570,1457,995 . \mathrm{mp}=113-115^{\circ} \mathrm{C}$.

6-methoxy-2,7-dimethyl-5,6,7,8-tetrahydro-1,6-naphthyridine (4h). Following the general procedure D from 5-methyl-3-(prop-1-en-1-yl)-1,2,4-triazine 1 h $(28 \mathrm{mg}, 0.2 \mathrm{mmol})$ and $\mathrm{N}$ methoxypropargylamine $2 \mathrm{~g}$ ( $27 \mu \mathrm{L}, 0.31 \mathrm{mmol}, 1.5$ equiv) for 48 hours at room temperature (TLC monitoring in 7:3 petroleum ether/EtOAc), the title compound was obtained as a brown oil (21 mg, 55\% yield) after silica gel column chromatography (eluent $=1: 1$ petroleum ether/EtOAc, $\left.R_{f}=0.2\right) .{ }^{1} \mathrm{H}$ NMR $\left(250 \mathrm{MHz}\right.$, dimethylsulfoxide- $\left.d_{6}, 80{ }^{\circ} \mathrm{C}\right): \delta_{\mathrm{H}} 7.34(\mathrm{~d}, J=7.8$ Hz, 1H,), 6.99 (d, $J=7.8 \mathrm{~Hz}, 1 \mathrm{H}), 4.22(\mathrm{~d}, J=15.7 \mathrm{~Hz}, 1 \mathrm{H}), 3.89$ (d, $J=15.7 \mathrm{~Hz}, 1 \mathrm{H}), 3.52$ (s, 3H), $3.27-3.02(\mathrm{~m}, 1 \mathrm{H}), 2.84(\mathrm{dd}, J=17.3,5.0 \mathrm{~Hz}, 1 \mathrm{H}), 2.68(\mathrm{dd}, J=17.3,9.5 \mathrm{~Hz}, 1 \mathrm{H})$, $2.39(\mathrm{~s}, 3 \mathrm{H}), 1.23(\mathrm{~d}, J=6.3 \mathrm{~Hz}, 3 \mathrm{H}) .{ }^{13} \mathrm{C}\left\{{ }^{1} \mathrm{H}\right\} \mathrm{NMR}\left(63 \mathrm{MHz}\right.$, dimethylsulfoxide- $\left.d_{6}, 80{ }^{\circ} \mathrm{C}\right)$ : 
$\delta(\mathrm{ppm})=155.0(\mathrm{C}), 152.6(\mathrm{C}), 134.2(\mathrm{CH}), 124.8(\mathrm{C}), 120.0(\mathrm{CH}), 59.8\left(\mathrm{CH}_{3}\right), 56.2\left(\mathrm{CH}_{2}\right)$, $53.8(\mathrm{CH}), \quad 36.4\left(\mathrm{CH}_{2}\right), 23.2\left(\mathrm{CH}_{3}\right), 17.9\left(\mathrm{CH}_{3}\right) . \quad \mathrm{ESI}^{+}-\mathrm{HRAM}: \mathrm{m} / z$ calculated for $\left[\mathrm{C}_{11} \mathrm{H}_{17} \mathrm{~N}_{2} \mathrm{O}\right]^{+}\left([\mathrm{M}+\mathrm{H}]^{+}\right)$193.133540, found 193.133203. IR (neat): $v_{\max } 2935,2810,2359$, 1470, 1033.

\section{6-methoxy-7-methyl-2-(trifluoromethyl)-5,6,7,8-tetrahydro-1,6-naphthyridine}

(4i).

Following the general procedure D from 3-(prop-1-en-1-yl)-5-(trifluoromethyl)-1,2,4-triazine 1i $(57 \mathrm{mg}, 0.30 \mathrm{mmol})$ and $N$-methoxypropargylamine $2 \mathrm{~g}(40 \mu \mathrm{L}, 38 \mathrm{mg}, 0.45 \mathrm{mmol}, 1.5$ equiv) for 48 hours at room temperature (TLC monitoring in 95:5 petroleum ether/EtOAc), the title compound was obtained as a brown oil (69 $\mathrm{mg}, 93 \%$ yield) after silica gel column chromatography $\left(\right.$ eluent $=$ gradient from 9:1 to 8:2 pentane/EtOAc, $R_{f}(8: 2$ petr. eth./EtOAc $)=$ 0.5). ${ }^{1} \mathrm{H}$ NMR $\left(250 \mathrm{MHz}\right.$, dimethylsulfoxide- $\left.d_{6}, 80{ }^{\circ} \mathrm{C}\right): \delta_{\mathrm{H}} 7.78(\mathrm{~d}, J=7.7 \mathrm{~Hz}, 1 \mathrm{H}), 7.62(\mathrm{~d}, J$ $=7.9 \mathrm{~Hz}, 1 \mathrm{H}), 4.39(\mathrm{~d}, J=16.8 \mathrm{~Hz}, 1 \mathrm{H}), 4.08(\mathrm{~d}, J=16.8 \mathrm{~Hz}, 1 \mathrm{H}), 3.53(\mathrm{~s}, 3 \mathrm{H}), 3.29(\mathrm{dqd}, J$ $=9.7,6.4,5.1 \mathrm{~Hz}, 1 \mathrm{H}), 2.95(\mathrm{dd}, J=17.5,5.1 \mathrm{~Hz}, 1 \mathrm{H}), 2.80(\mathrm{dd}, J=17.5,9.7 \mathrm{~Hz}, 1 \mathrm{H}), 1.26$ $(\mathrm{d}, J=6.4 \mathrm{~Hz}, 3 \mathrm{H}) .{ }^{13} \mathrm{C}\left\{{ }^{1} \mathrm{H}\right\} \mathrm{NMR}\left(63 \mathrm{MHz}\right.$, dimethylsulfoxide- $\left.d_{6}, 80{ }^{\circ} \mathrm{C}\right): \delta_{\mathrm{C}} 155.0(\mathrm{C})$, $144.13\left(\mathrm{q},{ }^{2} J_{\mathrm{C}-\mathrm{F}}=33.6 \mathrm{~Hz}, \mathrm{C}\right), 135.6(\mathrm{CH}), 132.5\left(\mathrm{q},{ }^{5} J_{\mathrm{C}-\mathrm{F}}=1.2 \mathrm{~Hz}, \mathrm{C}\right), 121.3\left(\mathrm{q},{ }^{1} J_{\mathrm{C}-\mathrm{F}}=274.0\right.$ $\left.\mathrm{Hz}, \mathrm{CF}_{3}\right), 117.5\left(\mathrm{q},{ }^{3} \mathrm{~J}_{\mathrm{C}-\mathrm{F}}=2.9 \mathrm{~Hz}, \mathrm{CH}\right), 60.0\left(\mathrm{CH}_{3}\right), 55.6(\mathrm{CH}), 53.6\left(\mathrm{CH}_{2}\right), 35.1\left(\mathrm{CH}_{2}\right), 18.0$ $\left(\mathrm{CH}_{3}\right)$. ESI ${ }^{+}-\mathrm{HRAM}: m / z$ calculated for $\left[\mathrm{C}_{11} \mathrm{H}_{14} \mathrm{~F}_{3} \mathrm{~N}_{2} \mathrm{O}\right]^{+}\left([\mathrm{M}+\mathrm{H}]^{+}\right)$247.105274, found 247.105466. IR (neat): $v_{\max } 3187,3085,2974,2359,1686,1340,1127$.

6-methoxy-2,3,7-trimethyl-5,6,7,8-tetrahydro-1,6-naphthyridine (4j). Following the general procedure D from 5,6-dimethyl-3-(prop-1-en-1-yl)-1,2,4-triazine $\mathbf{1 j}$ (45 mg, 0.30 mmol) and $N$-methoxypropargylamine $2 \mathrm{~g}(40 \mu \mathrm{L}, 38 \mathrm{mg}, 0.45 \mathrm{mmol}, 1.5$ equiv) for 72 hours at room temperature (TLC monitoring in 1:1 petroleum ether/EtOAc), the title compound was obtained as a brown solid (52 mg, 83\% yield) after silica gel column chromatography (eluent $=1: 1$ petroleum ether/EtOAc, $\left.R_{f}=0.2\right) .{ }^{1} \mathrm{H}$ NMR $\left(250 \mathrm{MHz}\right.$, dimethylsulfoxide- $\left.d_{6}, 80{ }^{\circ} \mathrm{C}\right): \delta_{\mathrm{H}}$ $7.16(\mathrm{~s}, 1 \mathrm{H}), 4.18(\mathrm{~d}, J=15.6 \mathrm{~Hz}, 1 \mathrm{H}), 3.86(\mathrm{~d}, J=15.6 \mathrm{~Hz}, 1 \mathrm{H}), 3.52(\mathrm{~s}, 3 \mathrm{H}), 3.14(\mathrm{dqd}, J=$ 
9.5, 6.3, 5.0 Hz, 1H), $2.80(\mathrm{dd}, J=17.2,5.0 \mathrm{~Hz}, 1 \mathrm{H}), 2.64(\mathrm{dd}, J=17.2,9.5 \mathrm{~Hz}, 1 \mathrm{H}), 2.35(\mathrm{~s}$, 3H), 2.19 (s, 3H), $1.22(\mathrm{~d}, J=6.3 \mathrm{~Hz}, 3 \mathrm{H}) .{ }^{13} \mathrm{C}\left\{{ }^{1} \mathrm{H}\right\}$ NMR (63 MHz, dimethylsulfoxide- $d_{6}, 80$ $\left.{ }^{\circ} \mathrm{C}\right): \delta_{\mathrm{C}} 153.7(\mathrm{C}), 149.6(\mathrm{C}), 134.7(\mathrm{CH}), 127.8(\mathrm{C}), 125.1(\mathrm{C}), 59.8\left(\mathrm{CH}_{3}\right), 56.4(\mathrm{CH}), 53.6$ $\left(\mathrm{CH}_{2}\right), 36.0\left(\mathrm{CH}_{2}\right), 21.3\left(\mathrm{CH}_{3}\right), 17.9\left(\mathrm{CH}_{3}\right), 17.6\left(\mathrm{CH}_{3}\right)$. ESI ${ }^{+}-H R A M: m / z$ calculated for $\left[\mathrm{C}_{12} \mathrm{H}_{19} \mathrm{~N}_{2} \mathrm{O}\right]^{+}\left([\mathrm{M}+\mathrm{H}]^{+}\right)$207.149190, found 207.148912. IR (neat): $v_{\max }$ 2971, 2931, 2897, $1443,1195,1042 . m p=64-65^{\circ} \mathrm{C}$.

6-methoxy-7-methyl-5,6,7,8-tetrahydro-1,6-naphthyridine (4k). Following the general procedure D from 3-(prop-1-en-1-yl)-1,2,4-triazine $1 \mathrm{~m}(36 \mathrm{mg}, 0.30 \mathrm{mmol})$ and $\mathrm{N}$ methoxypropargylamine $2 \mathrm{~g}$ ( $40 \mu \mathrm{L}, 0.45 \mathrm{mmol}, 1.5$ equiv) for 5 days at room temperature (TLC monitoring in 7:3 petroleum ether/EtOAc), the title compound was obtained as a lightyellow oil (15 mg, $0.08 \mathrm{mmol}, 27 \%$ yield) after column chromatography (eluent = 1:1 pentane/EtOAc, $\left.R_{f}=0.3\right) .{ }^{1} \mathrm{H}$ NMR $\left(250 \mathrm{MHz}\right.$, dimethylsulfoxide- $\left.d_{6}, 80{ }^{\circ} \mathrm{C}\right): \delta_{\mathrm{H}} 8.38-8.28$ $(\mathrm{m}, 2 \mathrm{H}), 7.53-7.41(\mathrm{~m}, 1 \mathrm{H}), 7.20-7.07(\mathrm{~m}, 1 \mathrm{H}), 4.27(\mathrm{~d}, J=16.0 \mathrm{~Hz}, 1 \mathrm{H}), 3.96(\mathrm{~d}, J=16.0$ $\mathrm{Hz}, 1 \mathrm{H}), 3.53(\mathrm{~s}, 3 \mathrm{H}), 3.20(\mathrm{ddd}, J=9.6,6.3,5.0 \mathrm{~Hz}, 1 \mathrm{H}), 2.89(\mathrm{dd}, J=17.4,5.0 \mathrm{~Hz}, 1 \mathrm{H})$, $2.74(\mathrm{dd}, J=17.4,9.6 \mathrm{~Hz}, 1 \mathrm{H}), 1.25(\mathrm{~d}, J=6.3 \mathrm{~Hz}, 3 \mathrm{H}) .{ }^{13} \mathrm{C}\left\{{ }^{1} \mathrm{H}\right\} \mathrm{NMR}(63 \mathrm{MHz}$, dimethylsulfoxide- $\left.d_{6}, 80{ }^{\circ} \mathrm{C}\right): \delta_{\mathrm{C}} 153.6(\mathrm{C}), 146.9(\mathrm{CH}), 133.8(\mathrm{CH}), 128.0(\mathrm{C}), 120.7(\mathrm{CH})$, $59.8\left(\mathrm{CH}_{3}\right), 54.0(\mathrm{CH}), 18.0\left(\mathrm{CH}_{2}\right)$, no signal for $\mathrm{C}^{8}\left(\mathrm{CH}_{2}\right)$. ESI ${ }^{+}$-HRAM: $m / z$ calculated for $\left[\mathrm{C}_{10} \mathrm{H}_{15} \mathrm{~N}_{2} \mathrm{O}\right]^{+}\left([\mathrm{M}+\mathrm{H}]^{+}\right)$179.117890, found 179.117741. IR (neat): $v_{\max }$ 2969, 2934, 2896, 2360, 1446, 1032.

6-(benzyloxy)-7-methyl-2-phenyl-5,6,7,8-tetrahydro-1,6-naphthyridine (4l). Following the general procedure D from 5-phenyl-3-(prop-1-en-1-yl)-1,2,4-triazine $\mathbf{1 b}$ (59 mg, $0.30 \mathrm{mmol}$ ) and $O$-benzyl- $N$-propargylhydroxylamine $2 \mathbf{h}(73 \mathrm{mg}, 0.45 \mathrm{mmol}, 1.5$ equiv.) for 19 hours at room temperature (TLC monitoring in 4:1 petroleum ether/EtOAc), the title compound was obtained as a beige solid (30 mg, 30\% yield) after silica gel column chromatography (dry loading, eluent $=9: 1$ pentane/EtOAc, $\left.R_{f}=0.3\right) .{ }^{1} \mathrm{H}$ NMR $\left(250 \mathrm{MHz}\right.$, dimethylsulfoxide- $d_{6}, 80$ 
$\left.{ }^{\circ} \mathrm{C}\right): \delta_{\mathrm{H}} 8.08-7.95(\mathrm{~m}, 2 \mathrm{H}), 7.68(\mathrm{~d}, J=8.0 \mathrm{~Hz}, 1 \mathrm{H}), 7.55(\mathrm{dt}, J=8.0,0.9 \mathrm{~Hz}, 1 \mathrm{H}), 7.51-$ $7.24(\mathrm{~m}, 8 \mathrm{H}), 4.78(\mathrm{~s}, 2 \mathrm{H}), 4.31(\mathrm{~d}, J=16.2 \mathrm{~Hz}, 1 \mathrm{H}), 4.05(\mathrm{dd}, J=16.2,0.9 \mathrm{~Hz}, 1 \mathrm{H}), 3.40-$ $3.21(\mathrm{~m}, 1 \mathrm{H}), 3.00(\mathrm{dd}, J=17.5,5.1 \mathrm{~Hz}, 1 \mathrm{H}), 2.84(\mathrm{dd}, J=17.5,9.5 \mathrm{~Hz}, 1 \mathrm{H}), 1.29(\mathrm{~d}, J=6.4$ $\mathrm{Hz}, 3 \mathrm{H}) .{ }^{13} \mathrm{C}\left\{{ }^{1} \mathrm{H}\right\}$ NMR (63 MHz, dimethylsulfoxide- $\left.d_{6}, 80{ }^{\circ} \mathrm{C}\right): \delta_{\mathrm{C}} 153.8(\mathrm{C}), 153.3(\mathrm{C})$, $138.5(\mathrm{C}), 137.4(\mathrm{C}), 134.9(\mathrm{CH}), 128.2(\mathrm{C}), 128.10(2 \mathrm{CH}), 128.08(2 \mathrm{CH}), 127.7(2 \mathrm{CH})$, $127.2(\mathrm{CH}), 126.9(\mathrm{CH}), 126.0(2 \mathrm{CH}), 117.3(\mathrm{CH}), 74.5\left(\mathrm{CH}_{2}\right), 56.4(\mathrm{CH}), 54.5\left(\mathrm{CH}_{2}\right), 38.5$ $\left(\mathrm{CH}_{2}\right), 18.0\left(\mathrm{CH}_{3}\right)$. ESI ${ }^{+}-\mathrm{HRAM}: m / z$ calculated for $\left[\mathrm{C}_{22} \mathrm{H}_{23} \mathrm{~N}_{2} \mathrm{O}\right]^{+}\left([\mathrm{M}+\mathrm{H}]^{+}\right)$331.180490, found 331.180870. IR (neat): $v_{\max } 3060,2933,2872,1451,1023,694 . \mathrm{mp}=89-90{ }^{\circ} \mathrm{C}$.

General procedure E, Samarium iodide mediated N-O bond cleavage of 6methoxytetrahydro-1,6-naphthyridines (5). ${ }^{25}$ Samarium iodide $(0.1 \mathrm{M}$ solution in THF, 3.5 equiv) was added to the 6-methoxytetrahydro-1,6-naphthyridines 4 under argon atmosphere at room temperature. The dark-blue mixture was stirred at room temperature until complete conversion of starting material was observed by TLC. Then, an ammonia solution in methanol (1 M, 20 equiv) was added. After 30 minutes of stirring, the reaction medium was diluted with water and saturated with solid sodium thiosulfate. The mixture was subsequently extracted with diethyl ether, the combined organic layers were dried over magnesium sulfate and concentrated on rotary evaporator. The expected tetrahydro-1,6-naphthyridine $\mathbf{5}$ was purified by silica gel column chromatography.

2-Phenyl-5,6,7,8-tetrahydro-1,6-naphthyridine (5a). Following the general procedure E from 6-methoxy-2-phenyl-5,6,7,8-tetrahydro-1,6-naphthyridine 4a (120 mg, $0.5 \mathrm{mmol})$ for 4 hours, the title compound was obtained as a light-yellow oil (88 $\mathrm{mg}, 84 \%)$ after column chromatography (4:1 AcOEt/EtOH, $\left.R_{f}=0.2\right) .{ }^{1} \mathrm{H}$ NMR $(400 \mathrm{MHz}$, chloroform- $d): \delta_{\mathrm{H}} 8.04$ $7.81(\mathrm{~m}, 2 \mathrm{H}), 7.53-7.34(\mathrm{~m}, 5 \mathrm{H}), 4.05(\mathrm{~s}, 2 \mathrm{H}), 3.27(\mathrm{t}, J=6.0 \mathrm{~Hz}, 2 \mathrm{H}), 3.03(\mathrm{t}, J=6.0 \mathrm{~Hz}$, 2H), $2.28($ br s, $1 \mathrm{H}) ;{ }^{13} \mathrm{C}\left\{{ }^{1} \mathrm{H}\right\}$ NMR (100.7 MHz, chloroform- $d$ ): $\delta_{\mathrm{C}} 155.5(\mathrm{C}), 155.1(\mathrm{C})$, $139.8(\mathrm{C}), 134.8(\mathrm{CH}), 129.8(\mathrm{C}), 128.8(2 \mathrm{CH}), 128.7(\mathrm{CH}), 127.0(2 \mathrm{CH}), 118.3(\mathrm{CH}), 47.6$ 
$\left(\mathrm{CH}_{2}\right), 44.2\left(\mathrm{CH}_{2}\right), 33.0\left(\mathrm{CH}_{2}\right)$. ESI ${ }^{+}-\mathrm{HRAM}: \mathrm{m} / \mathrm{z}$ calculated for $\left[\mathrm{C}_{14} \mathrm{H}_{15} \mathrm{~N}_{2}\right]^{+}\left([\mathrm{M}+\mathrm{H}]^{+}\right)$ 211.122975, found 211.122710. IR (neat): $v_{\max } 3367,3036,2926,1636,1588,1253,1025$, $831,773,738$.

7-pentyl-2-phenyl-5,6,7,8-tetrahydro-1,6-naphthyridine (5b). Following the general procedure E from 6-methoxy-7-pentyl-2-phenyl-5,6,7,8-tetrahydro-1,6-naphthyridine 4e (93 $\mathrm{mg}, 0.5 \mathrm{mmol})$ for 10 hours, the title compound was obtained as a light-orange oil (65 $\mathrm{mg}$, $78 \%$ yield) after silica gel column chromatography (eluent $=$ EtOAc, $\left.R_{f}=0.2\right) .{ }^{1} \mathrm{H}$ NMR $(400$ $\mathrm{MHz}$, chloroform- $d): \delta_{\mathrm{H}} 7.99-7.90(\mathrm{~m}, 2 \mathrm{H}), 7.51-7.40(\mathrm{~m}, 3 \mathrm{H}), 7.42-7.33(\mathrm{~m}, 2 \mathrm{H}), 4.10$ $(\mathrm{d}, J=16.5 \mathrm{~Hz}, 1 \mathrm{H}), 4.06(\mathrm{~d}, J=16.5 \mathrm{~Hz}, 1 \mathrm{H}) 3.09(\mathrm{dd}, J=16.9,4.0 \mathrm{~Hz}, 1 \mathrm{H}), 2.98(\mathrm{ddd}, J=$ 10.5, 6.6, 4.0 Hz, 1H), $2.69(\mathrm{dd}, J=16.9,10.5 \mathrm{~Hz}, 1 \mathrm{H}), 1.83(\mathrm{~s}, 1 \mathrm{H}), 1.64-1.54(\mathrm{~m}, 2 \mathrm{H})$, $1.48(\mathrm{~m}, 2 \mathrm{H}), 1.35(\mathrm{~m}, 4 \mathrm{H}), 0.97-0.87(\mathrm{~m}, 3 \mathrm{H}) .{ }^{13} \mathrm{C}\left\{{ }^{1} \mathrm{H}\right\}$ NMR $(101 \mathrm{MHz}$, chloroform- $d): \delta_{\mathrm{C}}$ $155.5(\mathrm{C}), 155.4(\mathrm{C}), 139.8(\mathrm{C}), 134.5(\mathrm{CH}), 129.4(\mathrm{C}), 128.8(2 \mathrm{CH}), 128.7(\mathrm{CH}), 127.0(2$ $\mathrm{CH}), 118.2(\mathrm{CH}), 54.2(\mathrm{CH}), 47.6\left(\mathrm{CH}_{2}\right), 39.3\left(\mathrm{CH}_{2}\right), 36.8\left(\mathrm{CH}_{2}\right), 32.1\left(\mathrm{CH}_{2}\right), 25.7\left(\mathrm{CH}_{2}\right)$, $22.8\left(\mathrm{CH}_{2}\right), 14.2\left(\mathrm{CH}_{3}\right)$. ESI ${ }^{+}-\mathrm{HRAM}: \mathrm{m} / z$ calculated for $\left[\mathrm{C}_{19} \mathrm{H}_{25} \mathrm{~N}_{2}\right]^{+}\left([\mathrm{M}+\mathrm{H}]^{+}\right) 281.201225$, found 281.201334. IR (neat): $v_{\max } 2954,2925,2855,2360,1587,1458,692$.

7-methyl-2-(trifluoromethyl)-5,6,7,8-tetrahydro-1,6-naphthyridine (5c). Following the general procedure E from 6-methoxy-7-methyl-2-(trifluoromethyl)-5,6,7,8-tetrahydro-1,6naphthyridine $4 \mathbf{i}(62 \mathrm{mg}, 0.25 \mathrm{mmol})$ for 8 hours, the title compound was obtained as a yellow oil (38 mg, 70\% yield) after silica gel column chromatography (eluent $=4: 1 \mathrm{EtOAc} / \mathrm{EtOH}, R_{f}$ $=0.2) .{ }^{1} \mathrm{H}$ NMR $(400 \mathrm{MHz}$, chloroform- $d): \delta_{\mathrm{H}} 7.48(\mathrm{~d}, J=8.0 \mathrm{~Hz}, 1 \mathrm{H}), 7.43(\mathrm{~d}, J=7.9 \mathrm{~Hz}$, 1H), $4.16(\mathrm{~d}, J=17.5 \mathrm{~Hz}, 1 \mathrm{H}), 4.08(\mathrm{~d}, J=17.5 \mathrm{~Hz}, 1 \mathrm{H}), 3.17-3.08(\mathrm{~m}, 1 \mathrm{H}), 3.05(\mathrm{dd}, J=$ $17.4,3.9 \mathrm{~Hz}, 1 \mathrm{H}), 2.67(\mathrm{dd}, J=17.4,10.6 \mathrm{~Hz}, 1 \mathrm{H}), 1.80(\mathrm{~s}, 1 \mathrm{H}), 1.28(\mathrm{~d}, J=6.3 \mathrm{~Hz}, 3 \mathrm{H})$. ${ }^{13} \mathrm{C}\left\{{ }^{1} \mathrm{H}\right\}$ NMR (101 MHz, chloroform- $d$ ): $\delta_{\mathrm{C}} 156.6(\mathrm{C}), 146.1\left(\mathrm{q},{ }^{2} J_{\mathrm{C}-\mathrm{F}}=34.3 \mathrm{~Hz}, \mathrm{C}\right), 134.9$ $(\mathrm{CH}), 134.1(\mathrm{C}), 121.7\left(\mathrm{q},{ }^{1} J_{\mathrm{C}-\mathrm{F}}=274.0 \mathrm{~Hz}, \mathrm{CF}_{3}\right), 117.8\left(\mathrm{q},{ }^{3} J_{\mathrm{C}-\mathrm{F}}=2.8 \mathrm{~Hz}, \mathrm{CH}\right), 49.4(\mathrm{CH})$, $47.6\left(\mathrm{CH}_{2}\right), 40.5\left(\mathrm{CH}_{2}\right), 22.3\left(\mathrm{CH}_{3}\right)$. ESI ${ }^{+}$-HRAM: $m / z$ calculated for $\left[\mathrm{C}_{10} \mathrm{H}_{12} \mathrm{~F}_{3} \mathrm{~N}_{2}\right]^{+}\left([\mathrm{M}+\mathrm{H}]^{+}\right)$ 
217.094709, found 217.094581. IR (neat): $v_{\max } 3400,3297,2965,2932,1340,1128,1110$.

\section{Acknowledgment}

This work was partially supported by INSA Rouen, Rouen University, Orléans University, CNRS, EFRD and Labex SynOrg (ANR-11-LABX-0029), Labex IRON (ANR-11-LABX0018-01) région Normandie (CRUNCh network) and région Centre-Val de Loire.

\section{Supporting Information Available}

Supporting Information Available: general experimental information, copies of NMR spectra for all newly formed products and X-Ray crystal data for compounds $9 \mathbf{a}, \mathbf{9 b}$ and $9 \mathbf{d}$. This material is available free of charge via the Internet at http://pubs.acs.org.

\section{References}

(1) Blakemore, D. C.; Castro, L.; Churcher, I.; Rees, D. C.; Thomas, A. W.; Wilson, D. M.; Wood, A. Organic synthesis provides opportunities to transform drug discovery. Nat. Chem. 2018, 10, 383.

(2) (a) Taylor, R. D.; MacCoss, M.; Lawson, A. D. G. Rings in Drugs. J. Med. Chem. 2014, 57, 5845. (b) Ritchie, T. J.; Macdonald, S. J. F.; Peace, S.; Pickett, S. D.; Luscombe, C. $\mathrm{N}$. The developability of heteroaromatic and heteroaliphatic rings - do some have a better pedigree as potential drug molecules than others? MedChemComm 2012, 3, 1062. (c) Ritchie, T. J.; Macdonald, S. J. F.; Young, R. J.; Pickett, S. D. The impact of aromatic ring count on compound developability: further insights by examining carbo- and hetero-aromatic and aliphatic ring types. Drug Discovery Today 2011, 16, 164.

(3) (a) Hoegenauer, K.; Soldermann, N.; Zécri, F.; Strang, R. S.; Graveleau, N.; Wolf, R. M.; Cooke, N. G.; Smith, A. B.; Hollingworth, G. J.; Blanz, J.; Gutmann, S.; Rummel, G.; Littlewood-Evans, A.; Burkhart, C. Discovery of CDZ173 (Leniolisib), Representing a Structurally Novel Class of PI3K Delta-Selective Inhibitors. ACS Med. Chem. Lett. 2017, 8, 975. (b) Clohessy, T. A.; Roberts, A.; Manas, E. S.; Patel, V. K.; Anderson, N. A.; Watson, A. J. B. Chemoselective One-Pot Synthesis of Functionalized Amino-azaheterocycles Enabled by COware. Org. Lett. 2017, 19, 6368. (c) Ishikawa, M.; Hashimoto, Y. Improvement in Aqueous Solubility in Small Molecule Drug Discovery Programs by Disruption of Molecular Planarity and Symmetry. J. Med. Chem. 2011, 54, 1539.

(4) (a) Schneider, P.; Schneider, G. Privileged Structures Revisited. Angew. Chem. Int. Ed. 2017, 56, 7971. (b) Lovering, F.; Bikker, J.; Humblet, C. Escape from Flatland:

Increasing Saturation as an Approach to Improving Clinical Success. J. Med. Chem. 2009, 52, 6752. (c) Lovering, F. Escape from Flatland 2: complexity and promiscuity. MedChemComm 2013, 4, 515.

(5) (a) Best, D.; Lam, H. W. C=N-Containing Azaarenes as Activating Groups in Enantioselective Catalysis. J. Org. Chem. 2014, 79, 831. (b) For reviews on conjugated 
addition reaction to alkenylazaarenes, see: Klumpp, D. A. Conjugate Additions to VinylSubstituted Aromatic N-Heterocycles. Synlett 2012, 23, 1590.

(6) To the best of our knowledge only one study briefly mentioned the use of 3-vinyl1,2,4-triazine in an aza-Michael reaction: Badarau, E.; Bugno, R.; Suzenet, F.; Bojarski, A. J.; Finaru, A.-L.; Guillaumet, G. SAR studies on new bis-aryls 5-HT7 ligands: Synthesis and molecular modeling. Bioorg. Med. Chem. 2010, 18, 1958.

(7) (a) Raw, S. A.; Taylor, R. J. K. In Advances in Heterocyclic Chemistry; Alan, R. K., Ed.; Academic Press: 2010; Vol. Volume 100, p 75. (b) Foster, R. A. A.; Willis, M. C. Tandem inverse-electron-demand hetero-/retro-Diels-Alder reactions for aromatic nitrogen heterocycle synthesis. Chem. Soc. Rev. 2013, 42, 63. (c) Boger, D. L. Diels-alder reactions of azadienes. Tetrahedron 1983, 39, 2869. (d) Duret, G.; Le Fouler, V.; Bisseret, P.; Bizet, V.; Blanchard, N. Diels-Alder and Formal Diels-Alder Cycloaddition Reactions of Ynamines and Ynamides. Eur. J. Org. Chem. 2017, 2017, 6816.

(8) (a) Berthonneau, C.; Buttard, F.; Hiebel, M.-A.; Suzenet, F.; Brière, J.-F. Organocatalyzed Thia-Michael Addition and Sequential Inverse Electron Demanding DielsAlder Reaction to 3-Vinyl-1,2,4- triazine Platforms. Adv. Synth. Catal. 2017, 359, 4106. (b) Jouha, J.; Buttard, F.; Lorion, M.; Berthonneau, C.; Khouili, M.; Hiebel, M. A.; Guillaumet, G.; Brière, J. F.; Suzenet, F. Domino Aza-Michael-ih-Diels-Alder Reaction to Various 3Vinyl-1,2,4-triazines: Access to Polysubstituted Tetrahydro-1,6-naphthyridines. Org. Lett. 2017, 19, 4770. (c) Lorion, M.; Guillaumet, G.; Brière, J.-F.; Suzenet, F. Sequential Michael Addition and Enamine-Promoted Inverse Electron Demanding Diels-Alder Reaction upon 3Vinyl-1,2,4-triazine Platforms. Org. Lett. 2015, 17, 3154.

(9) (a) Vitaku, E.; Smith, D. T.; Njardarson, J. T. Analysis of the Structural Diversity, Substitution Patterns, and Frequency of Nitrogen Heterocycles among U.S. FDA Approved Pharmaceuticals. J. Med. Chem. 2014, 57, 10257. (b) Hill, M. D. Recent Strategies for the Synthesis of Pyridine Derivatives. Chem.-Eur. J. 2010, 16, 12052. (c) Allais, C.; Grassot, J.M.; Rodriguez, J.; Constantieux, T. Metal-Free Multicomponent Syntheses of Pyridines. Chem. Rev. 2014, 114, 10829.

(10) (a) Platform 1 affords a new manifold in the field of the intramolecular $i h \mathrm{DA} / r \mathrm{DA}$ reaction of 1,2,4-triazines towards the construction of original fused-pyridine derivatives; for representative examples, see: Seitz, G.; Dietrich, S. Intramolekulare[4+2]Cycloadditionen substituierter Triazine mit inversem Elektronenbedarf

Intramolecular[4+2]-Cycloadditions of Substituted Triazines with Inverse Electron Demand. Archiv der Pharmazie 1984, 317, 379. (b) Taylor, E. C.; Macor, J. E. Novel intramolecular diels-alder reactions with alkynylthio derivatives of 1,2,4-triazines. New routes to thieno[2,3b]pyridines and thieno[2,3-c]pyridines. Tetrahedron Lett. 1985, 26, 2419. (c) Taylor, E. C.; Pont, J. L. Further intramolecular diels-alder reactions of 1,2,4-triazines. Synthesis of dihydropyrrolo[2,3-b]pyridines. Tetrahedron Lett. 1987, 28, 379. (d) Taylor, E. C.; Macor, J. E.; French, L. G. Intramolecular Diels-Alder reactions of 1,2,4-triazines. Synthesis of 2,3cyclopentenopyridines and 5,6,7,8-tetrahydroquinolines. J. Org. Chem. 1991, 56, 1807. (e) Haenel, F.; John, R.; Seitz, G. Trifluormethyl-substituierte, heterocyclisch anellierte Pyridine durch intramolekulare Diels-Alder-Cycloaddition mit inversem Elektronenbedarf. Archiv der Pharmazie 1992, 325, 349. (f) Branowska, D.; Ostrowski, S.; Rykowski, A. Tandem Vicarious Nucleophilic Substitution of Hydrogen/Intramolecular Diels\&ndash;Alder Reaction of 1,2,4-Triazines into Functionalized Cycloalkenopyridines. Chem. Pharm. Bull. 2002, 50, 463. (g) Diring, S.; Retailleau, P.; Ziessel, R. A Rational Protocol for the Synthesis of Arylated Bipyridine Ligands via a Cycloaddition Pathway. J. Org. Chem. 2007, 72, 10181. (h) Hajbi, Y.; Suzenet, F.; Khouili, M.; Lazar, S.; Guillaumet, G. Polysubstituted 2,3- 
dihydrofuro[2,3-b]pyridines and 3,4-dihydro-2H-pyrano[2,3-b]pyridines via microwaveactivated inverse electron demand Diels-Alder reactions. Tetrahedron 2007, 63, 8286. (i) Akritopoulou-Zanze, I.; Wang, Y.; Zhao, H.; Djuric, S. W. Synthesis of substituted fused pyridines, pyrazines and pyrimidines by sequential Ugi/inverse electron demand Diels-Alder transformations. Tetrahedron Lett. 2009, 50, 5773. (j) Hajbi, Y.; Suzenet, F.; Khouili, M.; Lazar, S.; Guillaumet, G. A Straightforward Approach to Substituted 2-(Hydroxymethyl)-2,3dihydrofuro[2,3-b]pyridines and 3-Hydroxy-3,4-dihydro-2H-pyrano[2,3-b]pyridines. Synthesis 2010, 1349. (k) Ma, Z.; Ni, F.; Woo, G. H. C.; Lo, S. M.; Roveto, P. M.; Schaus, S. E.; Snyder, J. K. An intramolecular inverse electron demand Diels-Alder approach to annulated alpha-carbolines. Beilstein J Org Chem 2012, 8, 829. (1) Dinh Ngoc, T.; Moons, N.; Kim, Y.; De Borggraeve, W.; Mashentseva, A.; Andrei, G.; Snoeck, R.; Balzarini, J.; Dehaen, W. Synthesis of triterpenoid triazine derivatives from allobetulone and betulonic acid with biological activities. Bioorg. Med. Chem. 2014, 22, 3292. (m) Bachollet, S. P. J. T.; Vivat, J. F.; Cocker, D. C.; Adams, H.; Harrity, J. P. A. Development of a Mild and Versatile Directed Cycloaddition Approach to Pyridines. Chem.-Eur. J. 2014, 20, 12889. (n) Bachollet, S. P. J. T.; Volz, D.; Fiser, B.; Münch, S.; Rönicke, F.; Carrillo, J.; Adams, H.; Schepers, U.; GómezBengoa, E.; Bräse, S.; Harrity, J. P. A. A Modular Class of Fluorescent Difluoroboranes: Synthesis, Structure, Optical Properties, Theoretical Calculations and Applications for Biological Imaging. Chem.-Eur. J. 2016, 22, 12430.

(11) For a recent alternative approach to fused-pyridines, see: Xie, L.-G.; Shaaban, S.; Chen, X.; Maulide, N. Metal-Free Synthesis of Highly Substituted Pyridines by Formal [2+2+2] Cycloaddition under Mild Conditions. Angew. Chem. Int. Ed. 2016, 55, 12864 and references cited therein.

(12) For representative examples, see: (a) Shah, U.; Lankin, C. M.; Boyle, C. D.; Chackalamannil, S.; Greenlee, W. J.; Neustadt, B. R.; Cohen-Williams, M. E.; Higgins, G. A.; Ng, K.; Varty, G. B.; Zhang, H.; Lachowicz, J. E. Design, synthesis, and evaluation of fused heterocyclic analogs of SCH 58261 as adenosine A2A receptor antagonists. Bioorg. Med. Chem. Lett. 2008, 18, 4204. (b) Kono, M.; Ochida, A.; Oda, T.; Imada, T.; Banno, Y.; Taya, N.; Masada, S.; Kawamoto, T.; Yonemori, K.; Nara, Y.; Fukase, Y.; Yukawa, T.; Tokuhara, H.; Skene, R.; Sang, B.-C.; Hoffman, I. D.; Snell, G. P.; Uga, K.; Shibata, A.; Igaki, K.; Nakamura, Y.; Nakagawa, H.; Tsuchimori, N.; Yamasaki, M.; Shirai, J.; Yamamoto, S. Discovery of [cis-3-(\{(5R)-5-[(7-Fluoro-1,1-dimethyl-2,3-dihydro-1H-inden-5yl)carbamoyl]-2-methoxy-7,8-dihydro-1,6-naphthyridin-6(5H)-yl \} carbonyl)cyclobutyl]acetic Acid (TAK-828F) as a Potent, Selective, and Orally Available Novel Retinoic Acid ReceptorRelated Orphan Receptor $\gamma \mathrm{t}$ Inverse Agonist. J. Med. Chem. 2018, 61, 2973. (c) Johnson, R. J.; O'Mahony, D. J. R.; Edwards, W. T.; Duncton, M. A. J. A concise one-pot synthesis of trifluoromethyl-containing 2,6-disubstituted 5,6,7,8-tetrahydroquinolines and 5,6,7,8tetrahydronaphthyridines. Org. Biomol. Chem. 2013, 11, 1358. (d) Jackl, M. K.; Kreituss, I.; Bode, J. W. Synthesis of Tetrahydronaphthyridines from Aldehydes and HARP Reagents via Radical Pictet-Spengler Reactions. Org. Lett. 2016, 18, 1713.

(13) (a) Tahirovic, Y. A.; Truax, V. M.; Wilson, R. J.; Jecs, E.; Nguyen, H. H.; Miller, E. J.; Kim, M. B.; Kuo, K. M.; Wang, T.; Sum, C. S.; Cvijic, M. E.; Schroeder, G. M.; Wilson, L. J.; Liotta, D. C. Discovery of N-Alkyl Piperazine Side Chain Based CXCR4 Antagonists with Improved Drug-like Properties. ACS Med. Chem. Lett. 2018, 9, 446. (b) Yoshiizumi, K.; Yamamoto, M.; Miyasaka, T.; Ito, Y.; Kumihara, H.; Sawa, M.; Kiyoi, T.; Yamamoto, T.; Nakajima, F.; Hirayama, R.; Kondo, H.; Ishibushi, E.; Ohmoto, H.; Inoue, Y.; Yoshino, K. Synthesis and structure-Activity relationships of 5,6,7,8-Tetrahydropyrido[3,4b]pyrazine-based hydroxamic acids as HB-EGF shedding inhibitors. Bioorg. Med. Chem. 2003, 11, 433. (c) Luckhurst, C. A.; Stein, L. A.; Furber, M.; Webb, N.; Ratcliffe, M. J.; 
Allenby, G.; Botterell, S.; Tomlinson, W.; Martin, B.; Walding, A. Discovery of isoindoline and tetrahydroisoquinoline derivatives as potent, selective PPAR $\delta$ agonists. Bioorg. Med.

Chem. Lett. 2011, 21, 492. (d) For representative examples, see: Shiozawa, A.; Ichikawa, Y.; Komuro, C.; Kurashige, S.; Miyazaki, H.; Yamanaka, H.; Sakamoto, T. Antivertigo Agents. III. Synthesis of 5, 6, 7, 8-Tetrahydro-1, 6-naphthyridine Methyl Homologs. Chem. Pharm. Bull. 1984, 32, 2522. (e) Cruz, D.; Wang, Z.; Kibbie, J.; Modlin, R.; Kwon, O. Diversity through phosphine catalysis identifies octahydro-1,6-naphthyridin-4-ones as activators of endothelium-driven immunity. Proc. Nalt. Acad. Sci. 2011, 108, 6769. (f) Harling, J. D.; Harrington, F. P.; Thompson, M. A facile synthesis of the 3-amini-5,6,7,8tetrahydro[1,6]naphtyridine systeme and some alkylated and polycyclic homologues. Synth. Commun. 2001, 31, 787. (g) Vanlaer, S.; Voet, A.; Gielens, C.; De Maeyer, M.; Compernolle, F. Bridged 5,6,7,8-Tetrahydro-1,6-naphthyridines, Analogues of Huperzine A: Synthesis, Modelling Studies and Evaluation as Inhibitors of Acetylcholinesterase. Eur. J. Org. Chem. 2009, 2009, 643.

(14) For leading examples of organocatalyzed conjugated addition reactions to alkenylazaarene, see: (a) Wang, S.; Li, X.; Liu, H.; Xu, L.; Zhuang, J.; Li, J.; Li, H.; Wang, W. Organocatalytic Enantioselective Direct Additions of Aldehydes to 4-Vinylpyridines and Electron-Deficient Vinylarenes and Their Synthetic Applications. J. Am. Chem. Soc. 2015, 137, 2303. (b) Hepburn, H. B.; Melchiorre, P. Brønsted acid-catalysed conjugate addition of photochemically generated [small alpha]-amino radicals to alkenylpyridines. Chem. Commun. 2016, 52, 3520. (c) Wang, Y.-Y.; Kanomata, K.; Korenaga, T.; Terada, M. Enantioselective Aza Michael-Type Addition to Alkenyl Benzimidazoles Catalyzed by a Chiral Phosphoric Acid. Angew. Chem. Int. Ed. 2016, 55, 927. (d) Yin, Y.; Dai, Y.; Jia, H.; Li, J.; Bu, L.; Qiao, B.; Zhao, X.; Jiang, Z. Conjugate Addition-Enantioselective Protonation of N-Aryl Glycines to $\alpha$-Branched 2-Vinylazaarenes via Cooperative Photoredox and Asymmetric Catalysis. $J$. Am. Chem. Soc. 2018, 140, 6083.

(15) For the alternative use of styrylisoxazoles as alkenylazaarene homologues, see: (a) Baschieri, A.; Bernardi, L.; Ricci, A.; Suresh, S.; Adamo, M. F. A. Catalytic Asymmetric Conjugate Addition of Nitroalkanes to 4-Nitro-5-styrylisoxazoles. Angew. Chem. Int. Ed. 2009, 48, 9342. (b) Sun, H.-W.; Liao, Y.-H.; Wu, Z.-J.; Wang, H.-Y.; Zhang, X.-M.; Yuan, W.-C. Enantioselective 1,6-Michael addition of anthrone to 3-methyl-4-nitro-5-alkenylisoxazoles catalyzed by bifunctional thiourea-tertiary amines. Tetrahedron 2011, 67, 3991. (c) Pei, Q.-L.; Sun, H.-W.; Wu, Z.-J.; Du, X.-L.; Zhang, X.-M.; Yuan, W.-C. Catalytic Asymmetric 1,6-Michael Addition of Arylthiols to 3-Methyl-4-nitro-5-alkenyl-isoxazoles with Bifunctional Catalysts. J. Org. Chem. 2011, 76, 7849.

(16) For a recent catalytic sulfa-Michael addition to alkenyl benzimidazoles, see: Formica, M.; Sorin, G.; Farley, A. J. M.; Diaz, J.; Paton, R. S.; Dixon, D. J. Bifunctional iminophosphorane catalysed enantioselective sulfa-Michael addition of alkyl thiols to alkenyl benzimidazoles. Chem Sci 2018, 9, 6969.

(17) (a) Kennedy, S. H.; Klumpp, D. A. Michael Additions Involving Amino Acid Esters with Alkenyl N-Heterocycles. J. Org. Chem. 2017, 82, 10219. (b) For an insightful pioneer investigation of aza-Michael reaction to vinylpyridine mentioning the use of metal or base/acid promoters, see: Reich, H. E.; Levine, R. The Pyridylethylation of Active Hydrogen Compounds. IV. The Acid-catalyzed Pyridylethylation of Primary Amines. J. Am. Chem. Soc. 1955, 77, 5434.

(18) (a) Han, X.; Civiello, R. L.; Mercer, S. E.; Macor, J. E.; Dubowchik, G. M. Syntheses of aza and fluorine-substituted 3-(piperidin-4-yl)-4,5-dihydro-1Hbenzo[d][1,3]diazepin-2(3H)-ones. Tetrahedron Lett. 2009, 50, 386. (b) Boy, K. M.; Guernon, J. M. Synthesis of 2-aminoethyl-5-carbethoxythiazoles utilizing a Michael-like addition 
strategy. Tetrahedron Lett. 2005, 46, 2251. (c) For representative examples of aza-Michael reaction to azaarenes, see: Cliffe, I. A.; Ifill, A. D.; Mansell, H. L.; Todd, R. S.; White, A. C. 7-amino-5,6,7,8-tetrahydroquinolines. Preparation from 5,6-dihydroquinoline and nitrogen nucleophiles. Tetrahedron Lett. 1991, 32, 6789. (d) Macor, J. E.; Ordway, T.; Smith, R. L.; Verhoest, P. R.; Mack, R. A. Synthesis and Use of 5-Vinyl-1,2,4-oxadiazoles as Michael Acceptors. A Rapid Synthesis of the Potent Muscarinic Agonist L-670,548. J. Org. Chem. 1996, 61, 3228.

(19) Xu, C.; Muir, C. W.; Leach, A. G.; Kennedy, A. R.; Watson, A. J. B. Catalytic Enantioselective Synthesis of alpha-Chiral Azaheteroaryl Ethylamines by Asymmetric Protonation. Angew. Chem. Int. Ed. 2018, 57, 11374.

(20) (a) Hui, C.; Pu, F.; Xu, J. Metal-Catalyzed Asymmetric Michael Addition in Natural Product Synthesis. Chem.-Eur. J. 2017, 23, 4023. (b) Amara, Z.; Caron, J.; Joseph, D. Recent contributions from the asymmetric aza-Michael reaction to alkaloids total synthesis. Nat. Prod. Rep. 2013, 30, 1211. (c) Howell, G. P. Asymmetric and Diastereoselective Conjugate Addition Reactions: C-C Bond Formation at Large Scale. Org. Proc. Res. Dev. 2012, 16, 1258. (d) For selected reviews, see: Zhang, Y.; Wang, W. Recent advances in organocatalytic asymmetric Michael reactions. Catal. Sci. Technol. 2012, 2, 42.

(21) Zhao, W.; Sun, J. Triflimide (HNTf2) in Organic Synthesis. Chem. Rev. 2018, $118,10349$.

(22) As first hypothesis, from either a thermodynamic point of view or for the ability to activate the vinyl moiety, the protonation of $\mathrm{N}^{2}$ of the vinyltriazines might be favored: Mó, O.; De Paz, J. L. G.; Yáñez, M. Protonation of azines: An ab initio molecular orbital study. Journal of Molecular Structure: THEOCHEM 1987, 150, 135.

(23) Liebeskind, L. S.; Srogl, J. Thiol Ester-Boronic Acid Coupling. A Mechanistically Unprecedented and General Ketone Synthesis. J. Am. Chem. Soc. 2000, 122, 11260 .

(24) (a) Alphonse, F.-A.; Suzenet, F.; Keromnes, A.; Lebret, B.; Guillaumet, G. Palladium-catalyzed 3-Thiomethyltriazine-boronic Acid Cross Coupling: Easy Access to 3Substituted-1,2,4-triazines. Synlett 2002, 447. (b) Alphonse, F.-A.; Suzenet, F.; Keromnes, A.; Lebret, B.; Guillaumet, G. Copper(I)-Promoted Palladium-Catalyzed Cross-Coupling of Unsaturated Tri-n-butylstannane with Heteroaromatic Thioether. Org. Lett. 2003, 5, 803.

(25) Revuelta, J.; Cicchi, S.; Brandi, A. Samarium(II) iodide reduction of isoxazolidines. Tetrahedron Lett. 2004, 45, 8375.

(26) Shi, D.-H.; Harjani, J. R.; Gable, R. W.; Baell, J. B. Synthesis of 3-(Alkylamino), 3-(Alkoxy)-, 3-(Aryloxy)-, 3-(Alkylthio)-, and 3-(Arylthio)-1,2,4-triazines by Using a Unified Route with 3-(Methylsulfonyl)-1,2,4-triazine. Eur. J. Org. Chem. 2016, 2016, 2842.

(27) Gantt, R. W.; Goff, R. D.; Williams, G. J.; Thorson, J. S. Probing the Aglycon Promiscuity of an Engineered Glycosyltransferase. Angew. Chem. Int. Ed. 2008, 47, 8889.

(28) Shrikhande, J. J.; Gawande, M. B.; Jayaram, R. V. A catalyst-free Nbenzyloxycarbonylation of amines in aqueous micellar media at room temperature. Tetrahedron Lett. 2008, 49, 4799.

(29) Gros, G.; Martinez, L.; Gimenez, A. S.; Adler, P.; Maurin, P.; Wolkowicz, R.; Falson, P.; Hasserodt, J. Modular construction of quaternary hemiaminal-based inhibitor candidates and their in cellulo assessment with HIV-1 protease. Bioorg. Med. Chem. 2013, 21, 5407.

(30) Wang, Y. D.; Kimball, G.; Prashad, A. S.; Wang, Y. Zr-Mediated hydroboration: stereoselective synthesis of vinyl boronic esters. Tetrahedron Lett. 2005, 46, 8777.

(31) Miyabe, H.; Asada, R.; Takemoto, Y. Cascade radical reaction of substrates with a carbon-carbon triple bond as a radical acceptor. Beilstein J. Org. Chem. 2013, 9, 1148. 
(32) Cross, B. P.; Schleich, T. Temperature dependence of the chemical shifts of commonly employed proton n.m.r. reference compounds. Org. Magn. Reson. 1977, 10, 82. 